\title{
Kv4.1, a Key Ion Channel For Low Frequency Firing of Dentate Granule Cells, Is Crucial for Pattern Separation
}

\author{
Kyung-Ran Kim, ${ }^{1 \star}$ Seung Yeon Lee, ${ }^{1,3 \star}$ Sang Ho Yoon, ${ }^{2,3}$ Yoonsub Kim, ${ }^{1,3}$ Hyeon-Ju Jeong, ${ }^{4}$ Sanghyeon Lee, ${ }^{3}$ \\ Young Ho Suh, ${ }^{2,3}$ Jong-Sun Kang, ${ }^{4}$ Hana Cho, ${ }^{5}$ Suk-Ho Lee, ${ }^{1,2,3}$ Myoung-Hwan Kim, ${ }^{1,2,3}$ and ${ }^{\odot W o n-K y u n g ~ H o ~}{ }^{1,2,3}$ \\ ${ }^{1}$ Department of Physiology, ${ }^{2}$ Neuroscience Research Institute, ${ }^{3}$ Department of Biomedical Science, Seoul National University College of Medicine, Seoul \\ 03080, Korea, ${ }^{4}$ Department of Molecular Cell Biology, and ${ }^{5}$ Department of Physiology, Sungkyunkwan University School of Medicine, Suwon 16419, Korea
}

The dentate gyrus (DG) in the hippocampus may play key roles in remembering distinct episodes through pattern separation, which may be subserved by the sparse firing properties of granule cells (GCs) in the DG. Low intrinsic excitability is characteristic of mature GCs, but ion channel mechanisms are not fully understood. Here, we investigated ionic channel mechanisms for firing frequency regulation in hippocampal GCs using male and female mice, and identified Kv4.1 as a key player. Immunofluorescence analysis showed that Kv4.1 was preferentially expressed in the DG, and its expression level determined by Western blot analysis was higher at 8-week than 3-week-old mice, suggesting a developmental regulation of Kv4.1 expression. With respect to firing frequency, GCs are categorized into two distinctive groups: low-frequency (LF) and high-frequency (HF) firing GCs. Input resistance $\left(R_{\mathrm{in}}\right)$ of most LF-GCs is lower than $200 \mathrm{M} \Omega$, suggesting that LF-GCs are fully mature GCs. Kv4.1 channel inhibition by intracellular perfusion of Kv4.1 antibody increased firing rates and gain of the input-output relationship selectively in LF-GCs with no significant effect on resting membrane potential and $R_{\text {in }}$, but had no effect in HF-GCs. Importantly, mature GCs from mice depleted of Kv4.1 transcripts in the DG showed increased firing frequency, and these mice showed an impairment in contextual discrimination task. Our findings suggest that Kv4.1 expression occurring at late stage of GC maturation is essential for low excitability of DG networks and thereby contributes to pattern separation.

Key words: dentate gyrus; Kv4.1; low excitability; mature granule cell; pattern separation

\section{Significance Statement}

The sparse activity of dentate granule cells (GCs), which is essential for pattern separation, is supported by high inhibitory inputs and low intrinsic excitability of GCs. Low excitability of GCs is thought to be attributable to a high $\mathrm{K}^{+}$conductance at resting membrane potentials, but this study identifies Kv4.1, a depolarization-activated $\mathrm{K}^{+}$channel, as a key ion channel that regulates firing of GCs without affecting resting membrane potentials. Kv4.1 expression is developmentally regulated and Kv4.1 currents are detected only in mature GCs that show low-frequency firing, but not in less mature high-frequency firing GCs. Furthermore, mice depleted of Kv4.1 transcripts in the dentate gyrus show impaired pattern separation, suggesting that Kv4.1 is crucial for sparse coding and pattern separation.

\section{Introduction}

Learning similar events rapidly as distinct episodic memories and retrieving them as such, a computational process called "pattern

\footnotetext{
Received June 10, 2019; revised Dec. 30, 2019; accepted Jan. 23, 2020.

Author contributions: K.-R.K., S.-H.L., and W.-K.H. designed research; K.-R.K., S.Y.L., S.H.Y., Y.K., H.-J.J., S.L., Y.H.S., J.-S.K., and H.C. performed research; K.-R.K., S.Y.L., S.H.Y., Y.K., H.-J.J., S.L., Y.H.S., J.-S.K., H.C., S.-H.L., M.-H.K., and W.-K.H. analyzed data; K.-R.K. wrote the first draft of the paper; S.-H.L., M.-H.K., and W.-K.H. wrote the paper.

This work was supported by the National Research Foundation of Korea (NRF Grants 2010-0027941 and 2017R1A2B2010186 to W.-K.H. and Grant 2017R1A6A3A11032599 to K.-R.K.) funded by the Korean Ministry of Science and ICT.

The authors declare no competing financial interests.

*K.-R.K. and S.Y.L. contributed equally to this work.

Correspondence should be addressed to Myoung-Hwan Kim at kmhwany@snu.ac.kr or Won-Kyung Ho at wonkyung@snu.ac.kr.
}

separation," are the hallmarks of hippocampal functions. It is postulated that the dentate gyrus (DG) of the hippocampus mediates pattern separation by transforming overlapping or perceptually similar sensory inputs into distinct neural representations (O'Reilly and McClelland, 1994; Treves and Rolls, 1994; Leutgeb et al., 2007). The sparse activity of granule cells (GCs), wherein only a few GCs are active within a relevant time window with low firing rates, has been regarded to be essential for the computational function to accomplish pattern separation (O'Reilly and McClelland, 1994; Treves and Rolls, 1994; Rolls, 2013).
https://doi.org/10.1523/JNEUROSCI.1541-19.2020

Copyright $\odot 2020$ the authors 
A hallmark of the DG is the continuous production of new neurons. During the period that newly generated GCs become fully mature to constitute a functionally homogenous neuronal population with developmentally born mature GCs (van Praag et al., 2002; Laplagne et al., 2006), the density of glutamatergic and GABAergic inputs and intrinsic excitability of GCs change enormously. Newly generated young GCs at age of 4-weeks display a low activation threshold due to high intrinsic excitability and enhanced excitation/inhibition balance, whereas mature GCs have a higher activation threshold due to strong inhibitory inputs and low intrinsic excitability (Schmidt-Hieber et al., 2004; Mongiat et al., 2009; Marín-Burgin et al., 2012; Dieni et al., 2013; Lopez-Rojas et al., 2016). However, how such different characteristics of young and mature GCs are relevant to DG functions is not fully understood. Most previous studies focused the role of adult-born young GCs in pattern separation (Clelland et al., 2009; Nakashiba et al., 2012), but it remains uncertain how the highly excitable young GCs can contribute to sparse and orthogonal dentate representation required for pattern separation function. The characteristic features of mature GCs, strong inhibitory inputs and low intrinsic excitability, may underlie sparse activity of GCs. Indeed, the hyperexcitability of GCs induced by selective degeneration of hilar mossy cells causing disinhibition led to impaired pattern separation (Jinde et al., 2012). However, whether low intrinsic excitability is critical for pattern separation has not been investigated. To directly test this possibility, identification of the mechanism specific to mature GCs' low excitability is prerequisite.

Kv4.2 and Kv4.3 are expressed abundantly in the brain (Baldwin et al., 1991; Pak et al., 1991; Serôdio et al., 1996), and encode A-type $\mathrm{K}^{+}$channels in association with $\mathrm{Kv}$ channel interacting proteins (Rhodes et al., 2004) playing a key role in the regulation of neuronal excitability in various brain regions including the hippocampus (Hoffman et al., 1997; Locke and Nerbonne, 1997; Shibata et al., 2000; Ramakers and Storm, 2002). Kv4.1, which belongs to Kv4 gene subfamily, is known to have similar electrophysiological and pharmacological properties when expressed in heterologous expression system (Baldwin et al., 1991; Pak et al., 1991; Serôdio et al., 1996), but its characteristics and roles in hippocampal neurons are largely unknown. In situ hybridization histochemistry showed that Kv4.1 signals are relatively strong in GCs compared with CA1 or CA3 pyramidal neurons (Serôdio and Rudy, 1998), suggesting a possibility that Kv4.1 may play a specific role in regulating excitability of GCs.

In the present study, we demonstrate that Kv4.1 is expressed preferentially in mature GCs with a subcellular distribution pattern distinctive from Kv4.2. With its unique electrophysiological properties distinct from classical $I_{\mathrm{A}}, \mathrm{Kv} 4.1$ contributes to the low firing rates of GCs without affecting their passive electrical properties. Furthermore, we show that selective Kv4.1 depletion in the DG leads to the impairment in contextual discrimination task, suggesting that the low rate firing of mature GCs is a key component required for pattern separation.

\section{Materials and Methods}

Animals. All experiments were performed with C57BL/6 mice. Animals were housed 3-5 mice per cage and maintained under specific pathogenfree (SPF) conditions with food and water freely available. Electrophysiological analyses for the dentate gyrus (DG) granule cells (GCs) were performed in 4- to 8-week-old mice of both sexes, whereas experiments for CA1 pyramidal cells (PCs) were conducted using 3- to 5-week-old mice. Electrophysiology experiments for the DG interneurons were performed in 7- to 8-week-old Vgat-ires-Cre mice (Slc32al ${ }^{\text {tm2(cre)Lowl }}$ knock-in; The Jackson Laboratory stock \#016962) infected with AAV2/ hSyn-DIO-mCherry. Behavioral analyses were performed with 10week-old male mice. The animal maintenance protocols and all experimental procedures were approved by the Institutional Animal Care and Use Committee at Seoul National University (SNU-090115-7, SNU-160825-1).

Slice electrophysiology. Mice were killed by decapitation after being anesthetized with isoflurane, and the whole brain was immediately removed from the skull, and chilled in artificial cerebrospinal fluid (aCSF) at $4^{\circ} \mathrm{C}$. Transverse hippocampal slices (350 $\mu \mathrm{m}$ thick) were prepared using a vibratome (VT1200S, Leica). Slices were incubated at $35^{\circ} \mathrm{C}$ for 30 min and thereafter maintained at $32^{\circ} \mathrm{C}$ until recording.

Hippocampal neurons were visualized using an upright microscope equipped with differential interference contrast (DIC) optics (BX51WI, Olympus). Electrophysiological recordings were made by the whole-cell patch-clamp technique with EPC-8 or EPC-10 amplifier (HEKA). Experiments were performed at $32 \pm 1{ }^{\circ} \mathrm{C}$. After membrane break-in, $2 \sim 3 \mathrm{~min}$ wait time was given to stabilize neurons. Patch pipettes with a tip resistance of 3.5-4 M $\Omega$ were used. Series resistance $\left(R_{\mathrm{s}}\right)$ after establishing whole-cell configuration was between 10 and $15 \mathrm{M} \Omega$. $R_{\mathrm{s}}$ was monitored by applying a short $(40 \mathrm{~ms})$ hyperpolarization $(5 \mathrm{mV})$ pulse during the recording. Cells were discarded when the $R_{\mathrm{s}}$ changed by $>20 \%$ of baseline value. The internal pipette solution contained the following (in $\mathrm{mm}$ ): 143 K-gluconate, $7 \mathrm{KCl}, 15 \mathrm{HEPES}, 4 \mathrm{MgATP}, 0.3 \mathrm{NaGTP}, 4 \mathrm{Na}-$ ascorbate, and 0.1 EGTA, with the $\mathrm{pH}$ adjusted to 7.3 with KOH. For the antibody-blocking experiments, the Kv4.1 antibody was included in the pipette solution $(0.3 \sim 1 \mu \mathrm{g} / \mathrm{ml})$. The bath solution (or aCSF) for control experiments contained the followings (in $\mathrm{mm}$ ): $125 \mathrm{NaCl}, 25 \mathrm{NaHCO}_{3}$, $2.5 \mathrm{KCl}, 1.25 \mathrm{NaH}_{2} \mathrm{PO}_{4}, 2 \mathrm{CaCl}_{2}, 1 \mathrm{MgCl}_{2}, 20$ glucose, 1.2 Na-pyruvate and $0.4 \mathrm{Na}$-ascorbate, $\mathrm{pH} 7.4$ when saturated with carbogen $\left(95 \% \mathrm{O}_{2}\right.$ and $5 \% \mathrm{CO}_{2}$ ). Under this experimental condition, calculated equilibrium potential for $\mathrm{K}^{+}$is $-107.4 \mathrm{mV}$.

For current-clamp experiments to analyze neuronal excitability, $20 \mu \mathrm{M}$ bicuculline and $10 \mu \mathrm{M}$ CNQX were included in bath solutions to block synaptic inputs, and the following parameters were measured: (1) resting membrane potential (RMP), (2) input resistance (membrane potential changes at a given hyperpolarizing current input ( $-35 \mathrm{pA}, 600 \mathrm{~ms})),(3)$ $F-I$ curve (firing frequencies (F) against the amplitude of injected currents (I)), (4) AP onset time (the delay from the start of the depolarized current injection to the beginning of the upstroke phase of the first evoked AP), (5) AP half-width (measured as the width at $50 \%$ of the spike peak amplitude), (6) Overshoot (difference in voltage of AP peak amplitude from $0 \mathrm{mV}$ ), (7) AP threshold (the voltage at the point of deflection for $\mathrm{d} V / \mathrm{d} t>40 \mathrm{mV} / \mathrm{ms}$ ). We recorded from cells with a wide range of RMP, but we did not adjust it to a fixed level by injecting currents when firing frequency or $R_{\text {in }}$ was measured. We applied step currents from the RMP to measure these parameters at their own RMP. Membrane potentials are given without correction for liquid junction potentials.

For voltage-clamp experiments to analyze outward $\mathrm{K}^{+}$currents, TTX $(0.5 \mu \mathrm{M}), \mathrm{CdCl}_{2}(300 \mu \mathrm{M})$, and $\mathrm{NiCl}_{2}(500 \mu \mathrm{M})$ were additionally applied to block $\mathrm{Na}^{+}$and $\mathrm{Ca}^{2+}$ channels, respectively. Outward $\mathrm{K}^{+}$currents were evoked by $1 \mathrm{~s}$ voltage steps to potentials between $-60 \mathrm{mV}$ to +50 $\mathrm{mV}$ from a holding potential of $-70 \mathrm{mV}$. After recording currents in the control condition, we added tetraethylammonium chloride (TEA) and 4-Aminopyridine (4-AP) sequentially for pharmacological dissection of $\mathrm{K}^{+}$currents. The difference currents between outward $\mathrm{K}^{+}$currents in control and those in $3 \mathrm{~mm}$ TEA were regarded as TEA-sensitive currents $\left(\mathrm{I}_{\mathrm{TEA}}\right)$, whereas the difference currents obtained after applying $5 \mathrm{~mm}$ 4-AP were regarded as 4-AP-sensitive currents $\left(I_{4-\mathrm{AP}}\right)$.

All chemicals were obtained from Sigma-Aldrich except CNQX, bicuculline, and TTX, which were from Abcam.

Immunofluorescence staining and Western blotting. For the immunofluorescence staining, C57BL/6 mice were killed at 3 (see Fig. 7) or 8 (see Fig. 1) weeks of age. AAV-injected mice were killed at 12 weeks of age. To generate Vgat-tdTomato mice, Vgat-ires-Cre mice were crossed with tdTo-

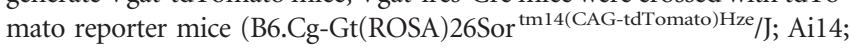
The Jackson Laboratory stock \#007914). Mice were anesthetized with isoflurane and perfused transcardially with a freshly prepared solution of $1 \times \mathrm{PBS}$ and $4 \%$ paraformaldehyde (PFA) for 10-15 min. Brains were 
removed and cut into $40 \mu \mathrm{m}$-thick sections using a vibratome (VT1200S, Leica), and postfixed overnight at $4^{\circ} \mathrm{C}$ by submersion in $4 \%$ PFA. After washing several times in $1 \times$ PBS with $0.3 \%$ Triton X-100 (PBS-T) for 5 min, sections were incubated 3 times in a blocking solution $(2.5 \%$ donkey serum $+2.5 \%$ goat serum or $5 \%$ donkey serum in $0.3 \%$ PBS-T) for $1 \mathrm{~h}$ at room temperature (RT). The sections were then incubated overnight at $4^{\circ} \mathrm{C}$ in blocking solutions containing the primary antibodies (anti-Kv4.1 antibody, APC-119, Alomone; anti-Kv4.2 antibody, APC023, Alomone; anti-doublecortin (DCX) antibody, C-18, Santa Cruz Biotechnology). After washing 5 times in $0.3 \%$ PBS-T for 5 min, sections were incubated with fluorescence-conjugated secondary antibodies diluted in blocking solution for $1 \mathrm{~h}$ at RT. Sections were washed 5 times in $0.3 \%$ PBS-T for $5 \mathrm{~min}$, and incubated with DAPI (Sigma-Aldrich) in PBS for 5 min. After rinsing with PBS, sections were mounted on glass slides using a fluorescent mounting medium (DakoCytomation). The immunostained sections were imaged with a confocal laser scanning microscope (FV1200, Olympus) using a $40 \times$ oil-immersion objective or $60 \times$ water-immersion objective, and then processed using Fluoview (Olympus).

For Western blotting, DG or CA1 region was isolated from slices under the dissecting microscope. Isolated tissues were homogenized with a glass homogenizer in TNE buffer (50 mM Tris-HCl, pH 8.0, $150 \mathrm{~mm} \mathrm{NaCl}$, and 2 mM EDTA) supplemented with protease inhibitor cocktails (Roche), and sonicated for $10 \mathrm{~s}$. After adding SDS (0.5\%) and Triton X-100 (1\%), lysates were incubated for $30 \mathrm{~min}$ at $4^{\circ} \mathrm{C}$. Insoluble materials were removed by centrifugation at $20,000 \times \mathrm{g}$ for $15 \mathrm{~min}$ at $4^{\circ} \mathrm{C}$. The amount of protein in the supernatants was determined by the Bradford assay, and supernatants were mixed with $6 \times$ Laemmli sample buffer. Samples containing $20 \mu \mathrm{g}$ of protein were loaded into each lane, separated by SDSPAGE, and transferred to a PVDF membrane. Membranes were blocked in $5 \%$ skim milk in TBS for $1 \mathrm{~h}$, and then probed with the relevant antibodies as indicated. The following antibodies were purchased from commercial sources: anti-Kv4.3, APC-017, Alomone; anti-Prox1 antibody, PRB-238C-200, BioLegend; anti- $\alpha$-tubulin, T5168, SigmaAldrich. Membranes were then incubated with peroxidase-conjugated secondary antibodies, and blots were detected with chemiluminescent reagents (Thermo Scientific).

HEK293 cell electrophysiology and immunocytochemistry. HEK293 cells were cultured in DMEM supplemented with $10 \%(\mathrm{v} / \mathrm{v})$ FBS and $1 \%(\mathrm{v} / \mathrm{v})$ penicillin/streptomycin in a humidified incubator supplied with $5 \% \mathrm{CO}_{2}$ at $37^{\circ} \mathrm{C}$. HEK 293 cells were plated in a 12 -well plate at a density of $1 \times 10^{5}$ or $0.5 \times 10^{5}$ cells per well for electrophysiology, and transfected with the $\mathrm{Kv} 4$ construct either alone ( Kv4.1 or Kv4.2) or together with a GFP construct (Kv4.3) using Lipofectamine 2000 (Thermo Scientific) at a ratio of 1:6 (DNA/lipid). The GFP-tagged Kv4.1 (catalog \#MG220056), GFP-tagged Kv4.2 (catalog \#MG209597) and Myc-tagged Kv4.3 (catalog \#MR221003) expression constructs were purchased from OriGene.

Transfected HEK293 cells were maintained in an incubator for 1-2 d for the expression of Kv4.1, Kv4.2 or Kv4.3, and then transferred to a recording chamber where bath solution was perfused at $1 \mathrm{ml} / \mathrm{min}$. The bath solution contained (in mM, $300 \pm 10 \mathrm{mOsm}$ ): $143 \mathrm{NaCl}, 5.4 \mathrm{KCl}, 5$ HEPES, $1.8 \mathrm{CaCl}_{2}, 0.5 \mathrm{MgCl}_{2}, 5$ glucose with $\mathrm{pH}$ adjusted to 7.4 with $\mathrm{NaOH} . \mathrm{K}^{+}$currents were recorded by whole-cell patch-clamp using a pipette solution of the following composition (in $\mathrm{mm}, 300 \pm 10 \mathrm{mOsm}$ ): $110 \mathrm{~K}$-aspartate, $30 \mathrm{KCl}, 10 \mathrm{HEPES}, 5 \mathrm{MgATP}, 1 \mathrm{MgCl}_{2}$, with the $\mathrm{pH}$ adjusted to 7.2 with $\mathrm{KOH}$ Experiments were performed at room temperature (RT). For antibody-blocking experiments, patch pipettes were filled with the internal solution containing the $\mathrm{Kv} 4.1$ antibody $(6 \mu \mathrm{g} / \mathrm{ml})$. To measure $\mathrm{K}^{+}$currents, we applied a $500 \mathrm{~ms}$ depolarizing voltage of $+40 \mathrm{mV}$ from a holding potential of $-80 \mathrm{mV}$. Patch pipette resistance and series resistance $\left(R_{\mathrm{s}}\right)$ after establishing whole-cell configuration were 3-4 $\mathrm{M} \Omega$ and 5-11 $\mathrm{M} \Omega$, respectively.

For immunocytochemistry, HEK293 cells were plated on poly-L-lysine (Sigma) coated coverslips in a 12-well plate and transfected with Kv4.1 or Kv4.2 cDNA. Cells were washed and fixed with $4 \%$ paraformaldehyde/4\% sucrose in PBS for $15 \mathrm{~min}$, and then permeabilized with $0.25 \%$ Triton X-100 for $5 \mathrm{~min}$. Afterward, they were blocked in PBS containing $1 \%$ normal goat serum for $1 \mathrm{~h}$ and incubated with primary antibody overnight at $4^{\circ} \mathrm{C}$. Coverslips were then incubated with Alexa 568- conjugated secondary antibody at RT for $1 \mathrm{~h}$ and mounted on slides. Images were captured using an Olympus FV 1000 laser scanning confocal microscope with a $63 \times$ objective.

AAV preparation. Short hairpin RNA (shRNA) cassettes targeting murine Kv4.1 sequence (GCTGCCTTCTGGTATACCATT) or containing a nontargeting control sequence (TCGCATAGCGTATGCCGTT) were cloned under mU6 promoter of pAAV-U6-GFP vector (Cell Biolabs). AAV particles were produced in HEK293T cells by cotransfecting pAAV$\mathrm{RC}$, pHelper, and rAAV vectors using calcium phosphate precipitation method. $72 \mathrm{~h}$ after transfection, cells were lysed by three times of thaw/ freeze cycles and the crude viral supernatant was obtained after a centrifugation at $12,000 \times g$ for $30 \mathrm{~min}$ at $4^{\circ} \mathrm{C}$. After the viral supernatant was overlaid on iodixanol (OptiPrep; Axis Shield) discontinuous density gradient (15/25/40/54\%), AAV particles were collected at a $40 \%$ iodixanol density layer following a centrifugation at $350,000 \times g$ for 90 min at $18^{\circ} \mathrm{C}$. AAV particles were dialyzed and concentrated using Amicon Ultra-15 centrifugal filter with Ultracel-100 membrane (Millipore). Aliquoted viral stock $\left(\sim 5 \times 10^{12} \mathrm{GC} / \mathrm{ml}\right)$ was stored at $-80^{\circ} \mathrm{C}$ before use. AAV2/ hSyn-DIO-mCherry (\#50459) was obtained from Addgene.

Stereotaxic surgery. Five-week-old mice were anesthetized and stereotaxically injected with high titers $\left(\sim 5 \times 10^{12} \mathrm{GC} / \mathrm{ml}\right)$ of AAVs $(1 \mu \mathrm{l})$. The injection was performed through glass capillary at 4 sites at the following coordinates relative to bregma $(\mathrm{mm})$ : AP: -2 , ML: $\pm 1.6, \mathrm{DV}:-2.5$; or AP: -3, ML: \pm 2.6, DV: -3.2 . After infusion, the glass capillary was left in place for an additional $5 \mathrm{~min}$ to ensure full virus diffusion. After surgery, mice were treated with antibiotics daily for 2 weeks, and their health was monitored every day.

Behavior analysis. contextual fear conditioning and discrimination task were performed 3 weeks after surgery. Mice were trained to discriminate between two similar contexts, A and B, through repeated experience in each context. Context A (conditioning context) was a chamber $(18 \mathrm{~cm}$ wide $\times 18 \mathrm{~cm}$ long $\times 30 \mathrm{~cm}$ high; H10-11M-TC; Coulbourn Instruments 5583, PA 18052) consisting of a metal grid floor, aluminum side walls, and a clear Plexiglas front door and back wall. context A was indirectly illuminated with a $12 \mathrm{~W}$ light bulb. The features of context $\mathrm{B}$ (safe context) were the same as context $\mathrm{A}$, except for a unique scent $(1 \%$ acetic acid), dimmer light ( $50 \%$ of $\mathrm{A}$ ), and a sloped floor by $15^{\circ}$ angle. Each context was cleaned with $70 \%$ ethanol before the animals were placed. On the first $3 \mathrm{~d}$ (contextual fear acquisition), mice were placed in context A for $3 \mathrm{~min}$ for exploring the environment, and then received a single foot shock $(0.75 \mathrm{~mA}$, for $2 \mathrm{~s})$. Mice were returned to their home cage 1 min after the shock. On days $4-5$, mice of each genotype were divided into two groups; one group visited context A on day 4 and context $\mathrm{B}$ on day 5 , whereas the other group visited the context $\mathrm{B}$ on day 4 and context A on day 5. On days 4-5 (generalization), neither group received a shock in context $\mathrm{A}$ or $\mathrm{B}$ and the freezing level was measured for 5 min only in context A. We defined freezing behavior as behavioral immobility except for respiration movement (McNaughton and Nadel, 1990). We observed video image for $2 \mathrm{~s}$ bouts every $10 \mathrm{~s}$ ( 18 or 30 observation bouts for $3 \mathrm{~min}$ or $5 \mathrm{~min}$ recording time) and counted the number of $2 \mathrm{~s}$ bouts during which the mouse displayed freezing behavior (referred to as the freezing score). The percentage of freezing was calculated by dividing the freezing score with the total number of observation bouts (18 or 30). Mice were subsequently trained to discriminate these two contexts by visiting the two contexts daily for $7 \mathrm{~d}$ (from day 6 to 12 , discrimination task). Mice always received a foot shock (2s) 3 min after being placed in context A but not B. Discrimination ratios were calculated according to $F_{\mathrm{A}} /\left(F_{\mathrm{A}}+F_{\mathrm{B}}\right)$, where $F_{\mathrm{A}}$ and $F_{\mathrm{B}}$ are freezing scores in contexts $A$ and $B$, respectively. All experiments and analyses were performed blind to the mice genotype.

Open field exploration test (OFT) was performed in white plastic boxes $(40 * 40 * 40 \mathrm{~cm})$ in a dimly lit room. The open field was divided into a center zone (center, $20 * 20 \mathrm{~cm}$ ) and an outer field. Individual mice were placed in the center zone, and the path of the animal was recorded with a video camera. Total distance traveled was measured for the entire period $(20 \mathrm{~min})$ and time spent in the center zone was analyzed for initial 5 min.

Elevated plus maze (EPM) test was performed using a plus-shaped apparatus consists of two open arms and two closed arms. Each arm was 
$65 * 6.25 \mathrm{~cm}$ in size, and the maze was elevated $50 \mathrm{~cm}$ above the ground. Mice were individually placed on the center area, facing an open arm, and allowed to freely explore the apparatus for $5 \mathrm{~min}$. We analyzed the total distance traveled for $5 \mathrm{~min}$ and time spent in each arm. OFT and EPM were analyzed using EthoVision XT (Noldus, Netherlands)

Novel object recognition tests were conducted in the same open field boxes used for the OFT. Two identical objects were placed in the box during the familiarization phase, and one of the objects was replaced with a similar new object during the discrimination test. The interval between the familiarization phase and the discrimination test was $24 \mathrm{~h}$. In each session, mice were allowed to explore freely the objects, and exploratory activity was recorded for $10 \mathrm{~min}$. Discrimination index was calculated as the time spent exploring a similar novel object divided by the total time spent exploring both the novel and the familiar objects.

Statistical analysis. Data were analyzed with Igor Pro (Version 6; Wavemetrics) and Origin (Version 8; Microcal). All results are presented as mean \pm SEM with the number of cells or mice $(n)$ used in each experiment. Statistical significance was evaluated using Student's $t$ test, and the level of significance was indicated by the number of marks $\left({ }^{\star} p<\right.$ $\left.0.05 ;{ }^{* *} p<0.01 ;{ }^{* *} p<0.001\right) . p>0.05$ was regarded as not significantly different (N.S.). Comparison between multifactorial statistical data was made using the two-way ANOVA. Differences in time-dependent changes of behavioral parameters between the two genotypes were evaluated using two-way repeated-measures ANOVA.

\section{Results}

\section{Preferential expression of Kv4.1 in the DG}

Among Kv4 family channels that mediate $I_{\mathrm{A}}, \mathrm{Kv} 4.2$ is highly expressed in principal neurons throughout the hippocampus and known to play crucial roles in regulating intrinsic excitability and synaptic plasticity (Kim et al., 2005, 2007; Chen et al., 2006). However, little is known about the distribution and functions of Kv4.1. We first investigated the distribution and expression of Kv4.1 using the Kv4.1-specific antibody in comparison with $\mathrm{Kv} 4.2$ in different subregions of the hippocampus obtained from adult mouse (8-week-old). Antibody specificity was confirmed using HEK293 cells expressing Kv4.1 or Kv4.2 isoform alone (Fig. $1 A)$. Immunohistochemistry and Western blot analyses showed that both Kv4.1 and Kv4.2 were expressed in the hippocampus, but their regional and subcellular distributions were different. Specifically, Kv4.1 signal was evident in the DG and CA3, but it was very weak in CA1, whereas Kv4.2 signal was strong both in the DG and CA1, but weak in CA3 (Fig. 1B). Moreover, the two isoforms showed distinct patterns of subcellular localization. Kv4.1 signals were localized mainly in the principal cell layer of the hippocampus, whereas Kv4.2 signals were localized mainly in dendritic regions (Fig. 1B). Average fluorescence intensities for Kv4.1 and Kv4.2 obtained from the DG and CA1 subregions and Western blot analysis confirmed these observations (Fig. 1C,D).

\section{Kv4.1 inhibition selectively increases the firing frequency of low-frequency-firing GCs}

To investigate the functional significance of Kv4.1 expression in GCs, we generated an adeno-associated virus (AAV) vector to stably express a short hairpin RNA (shRNA) against the Kv4.1 transcript (shKv4.1), and stereotaxically injected into the hippocampal DG area. Kv4.1-immunoreactive signals were not affected by infection of nontargeting shRNA sequence (shCtrl), but significantly reduced specifically in the region where shKv4.1 was infected (Fig. 2A). To further determine the specificity of shKv4.1, we expressed shKv4.1 in cultured hippocampal neurons. Similar to mice infected with shKv4.1, the infection of shKv4.1 significantly suppressed Kv4.1 expression in cultured hippocampal neurons, whereas the expression levels of both Kv4.2 and Kv4.3 were not affected by the shKv4.1 infection, con- firming the specificity and efficacy of shKv4.1 and Kv4.1 antibody (Fig. 2B). We then examined the effect of Kv4.1 knock-down on the electrical properties of GCs 2 weeks after stereotaxic injection of shKv4.1 or shCtrl into the DG. To avoid possible sources of confounding factors such as the neural progenitor cells and newborn immature neurons, we chose GCs in the outer granular cell layer (GCL) where mature GCs are located (Kerloch et al., 2019). When we induced repetitive APs using a depolarizing current injection, however, the firing rate was significantly higher in shKv4.1-infected GCs than in shCtrl-infected GCs (Fig. 2Ca). The relationships between the firing frequency and input current (F-I curves) shifted upward in shKv4.1-infected GCs with an increase in slope, indicating the increased gain of input-output relationship ( $I-O$ gain) (Fig. $2 C b$ ). $I-O$ gain calculated from the frequency increment between 100 and $200 \mathrm{pA}$ input current was significantly higher in shKv4.1-infected GCs $(20.2 \pm 1.7, n=9)$ than in shCtrl-infected GCs $(6.6 \pm 1.4, n=11, p<0.0001)$. Interestingly, resting membrane potential (RMP) and input resistance $\left(R_{\text {in }}\right)$ in shKv4.1-infected GCs and those in shCtrlinfected GCs showed no significant difference between groups ( $p=0.76$ for RMP; $p=0.72$ for $R_{\text {in }}$, Fig. $2 D$ ), and the values were consistent with those reported previously for mature GCs (Schmidt-Hieber et al., 2004; Dieni et al., 2013). These results suggest that Kv4.1 channels do not affect the passive membrane properties, but contribute to the low firing rate of GCs.

Electrical properties of GCs are not homogenous, since they change during maturation, in a way that $R_{\text {in }}$ decreases and RMP hyperpolarizes with time (Schmidt-Hieber et al., 2004; Mongiat et al., 2009; Dieni et al., 2013). To further study the role of Kv4.1 in firing frequency regulation, we first examined the heterogeneity of firing properties among GCs with $R_{\text {in }}$ between 100 and 600 $\mathrm{M} \Omega$ where depolarizing current injection evoked repetitive firing faithfully. Cells with $R_{\text {in }}$ in this range exhibited typical morphology of GCs (Fig. 3A), and GCs with lower $R_{\text {in }}$ were found in the outer granule cell layer (GCL), whereas GCs with higher $R_{\text {in }}$ were mostly found in the inner GCL. The relative positions of the cells shown in Figure $3 A$ in the GCL were $0.19,0.3$, and 0.69 when the GCL was scaled from 0 to 1 from the border of molecular layer to the hilus. RMP varied widely between -50 to $-95 \mathrm{mV}$, in a way that RMP hyperpolarization occurred steeply as $R_{\text {in }}$ decreased lower than $300 \mathrm{M} \Omega$ (Fig. 3B). The firing frequency of repetitive APs induced by long depolarization (1 s duration) also varied greatly ranging from a few to $>50$. Notably, the distribution of firing frequency was not as continuous as $R_{\text {in }}$ or RMP (Fig. $3 B$ ), but can be categorized into two distinctive groups (Fig. $3 C$ ). One group of GCs was hardly activated in response to a depolarizing pulse of $100 \mathrm{pA}$, and the number of APs induced by $200 \mathrm{pA}$ current injection was $<12$, whereas the other group of GCs was much more excitable, and the number of APs induced by $200 \mathrm{pA}$ current injection was far above 20. We therefore referred to the former group as the low-frequency-firing GCs (LF-GCs) and the latter group as the high-frequency-firing GCs (HF-GCs). The relationship between firing frequency and $R_{\mathrm{in}}$ or RMP showed a tendency that firing frequency increased with $R_{\text {in }}$ and RMP depolarization. Most LF-GCs had $R_{\text {in }}$ smaller than $200 \mathrm{M} \Omega$ and RMP lower than $-75 \mathrm{mV}$ (Fig. 3Cb, $C c$, blue symbols). The mean $R_{\text {in }}$ value for LF-GCs (153.6 $\left.\pm 6.6 \mathrm{M} \Omega, n=20\right)$ was significantly smaller than the value for HF-GCs $(376.8 \pm 21.3 \mathrm{M} \Omega, n=27$, $p<0.001)$, and the mean RMP value for LF-GCs $(-82.9 \pm 1.2$ $\mathrm{mV}, n=20$ ) was significantly lower than that for HF-GCs $(-64.1 \pm 1.8 \mathrm{mV}, n=27, p<0.001)$. In contrast, the properties of single AP, such as AP threshold, overshoot, and AP width, were fairly constant in GCs with $R_{\text {in }}$ in this range, and there was no 
A

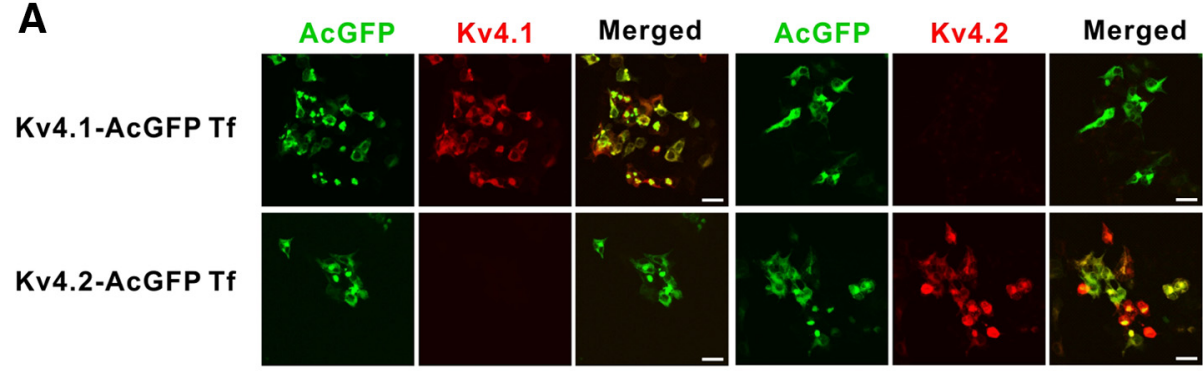

B
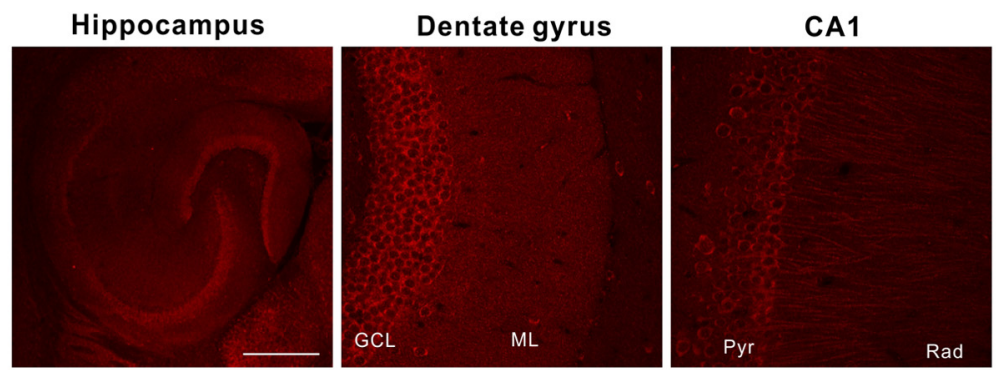

Kv4.1

Kv4.2
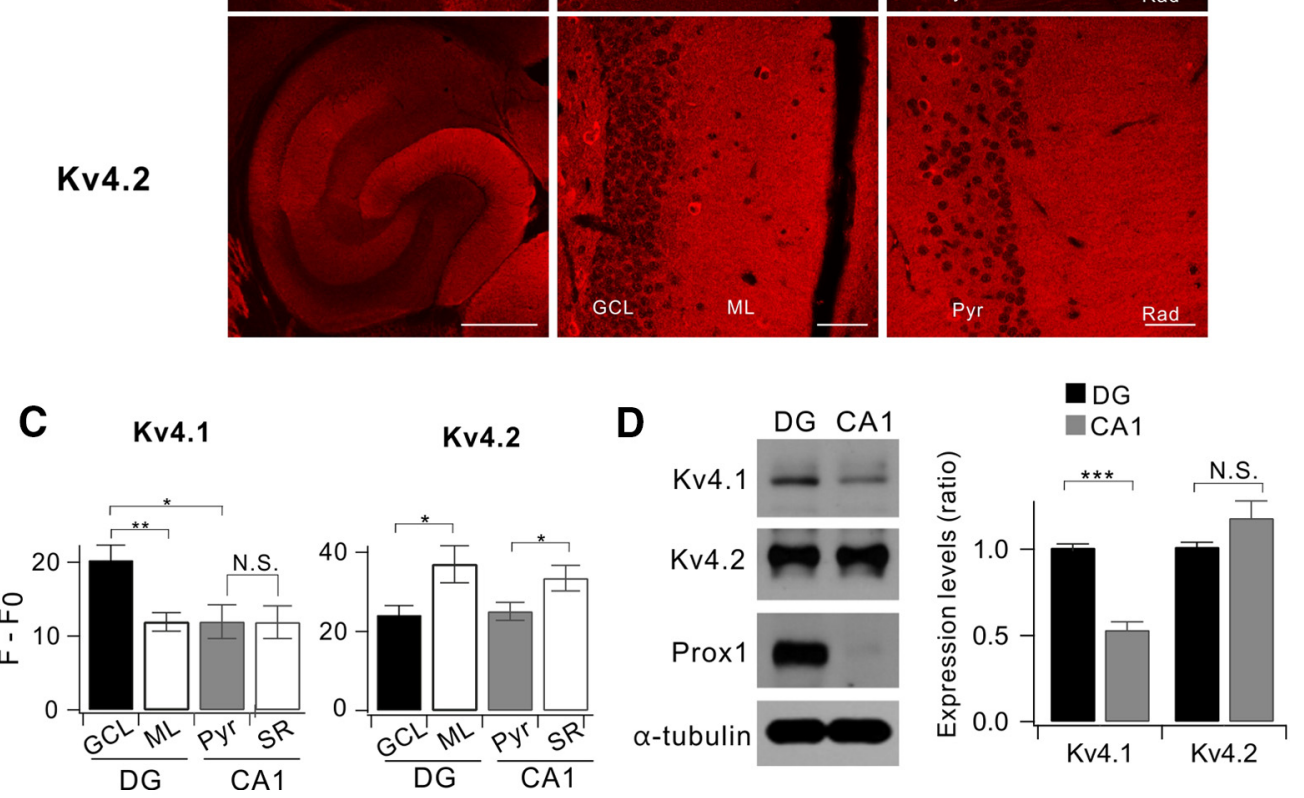

Figure 1. Comparison between Kv4.1 and Kv4.2 expression in the hippocampus. A, Both Kv4.1 and Kv4.2 antibodies selectively recognize the channel in HEK293 cells. HEK293 cells were transfected with either AcGFP-tagged Kv4.1 CDNA (Kv4.1-AcGFP Tf) or Kv4.2 (Kv4.2-AcGFP Tf). Forty-eight hours after transfection, the cells were stained using Kv4.1 or Kv4.2 antibodies. Scale bar, $20 \mu \mathrm{m} . \boldsymbol{B}$, Representative fluorescence immunostaining images show that Kv4.1 (top) is highly expressed in DG while Kv4.2 (bottom) is broadly expressed in the hippocampus of 8-week-old mice. Scale bars, $500 \mu \mathrm{m}$ (left) and $50 \mu \mathrm{m}$ (middle and right). C, Summary bar graphs of the relative fluorescence intensity $\left(F-F_{0}\right)$ shown in the DG and CA1 for Kv4.1 ( $n=10$, left) and Kv4.2 ( $n=5$, right). (Kv4.1;GCL, 20.3 $\pm 2.0 ; \mathrm{ML}, 11.9 \pm 1.3 ; \mathrm{Pyr}, 12.0 \pm 2.3 ; \mathrm{Rad}, 11.9 \pm 2.2 ; \mathrm{GCL}$ vs ML, $p=0.0037 ; \mathrm{GCL}$ vs Pyr, $p=0.013 ; \mathrm{Pyr}$ vs Rad, $p=0.97 ; \mathrm{Kv} 4.2 ; \mathrm{GCL}, 24.2 \pm 2.3 ; \mathrm{ML}, 39.1 \pm 5.8 ; \mathrm{Pyr}$, $25.1 \pm 2.3 ; \operatorname{Rad}, 33.5 \pm 3.3 ; G(L$ v ML, $p=0.037 ;$ Pyrvs Rad, $p=0.016$ ). D. Western blot analysis for Kv4.1 and Kv4.2 in DG and CA1 of 8-week-old mice. Right, DG exhibits higher Kv4.1 expression level compared with CA1; $1.00 \pm 0.02$ in DG, $0.53 \pm 0.05$ in CA1, $n=8 ; p<0.0001$. Paired $t$ test. Prospero-related homeobox 1 (Prox) and $\alpha$-tubulin used as a marker for DG and loading control, respectively. ${ }^{*} p<0.05,{ }^{* *} p<0.01,{ }^{* * *} p<0.001$, N.S. (not significant) $p>0.05$ by Student's t-test.

significant difference between LF-GCs and HF-GCs (Fig. 3D). These results suggest that ion channels required for single AP generation mature before $R_{\text {in }}$ reaches $\sim 600 \mathrm{M} \Omega$, whereas ion channels responsible for RMP hyperpolarization and lowering frequency mature at a late stage.

The difference in firing frequencies between LF-GCs and HFGCs was further analyzed in $F-I$ curves. The minimum current for activation was much larger, resulting in a rightward shift in the F-I curve in LF-GCs (Fig. 3E). Furthermore, the slope of the $F-I$ curve was less steep in LF-GCs, indicating that the $I-O$ gain was lower. $I-O$ gain was analyzed by comparing the frequency increment for a given increase in input current, showing that the input current range where the $I-O$ gain was maximum is different between LF-GCs and HF-GCs and that the maximum gain was significantly larger in HF-GCs $(-7.58 \pm 0.35, n=12$, between 200 and $250 \mathrm{pA}$ for LF-GCs; $-14.29 \pm 0.95, n=13$, between 50 and $100 \mathrm{pA}$ for HF-GCs, $p<0.001$, Fig. $3 F)$.

We then investigated whether Kv4.1 may have different roles in LF-GCs and HF-GCs. Since the Kv4.1 antibody used for immunohistochemistry was made against intracellular epitopes, we examined whether it can be used for the functional blockade of Kv4.1 channels. Application of the Kv4.1 antibody through a patch pipette robustly inhibited the Kv4.1 currents but not the Kv4.2 currents or Kv4.3 currents overexpressed in HEK293 cells 
A

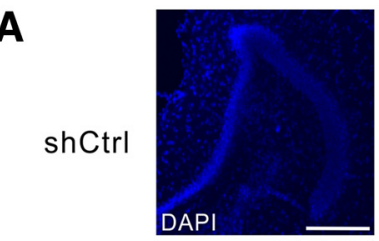

shKv4.1
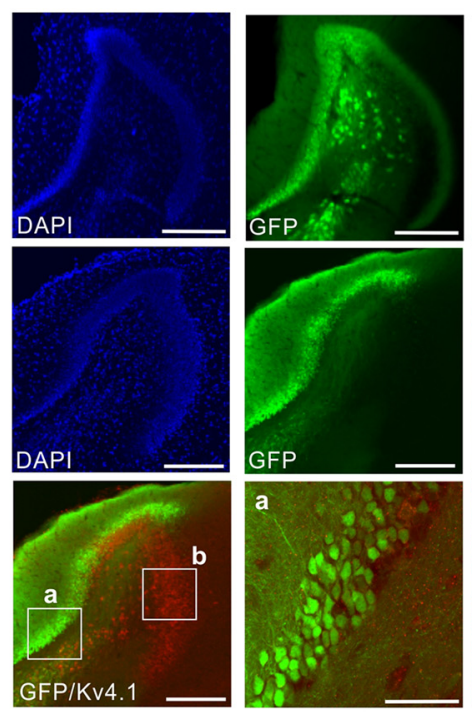
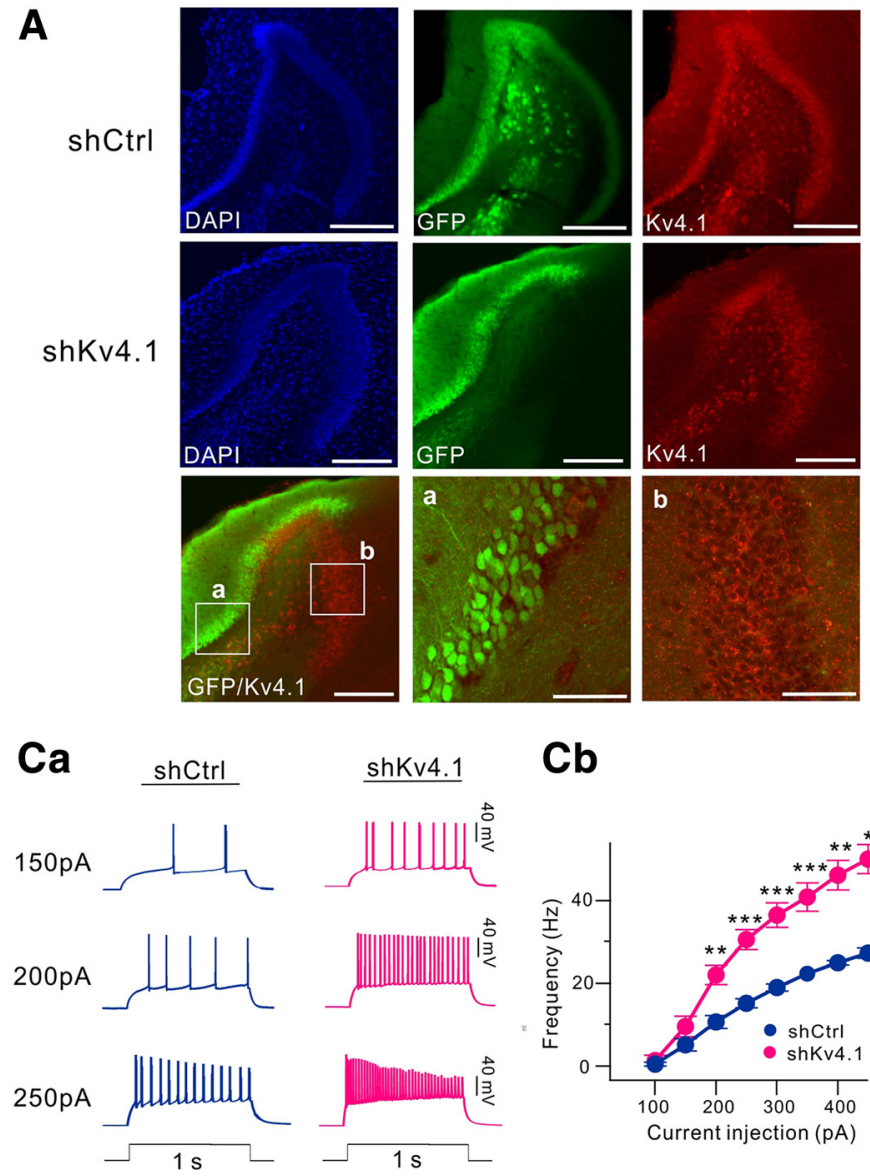
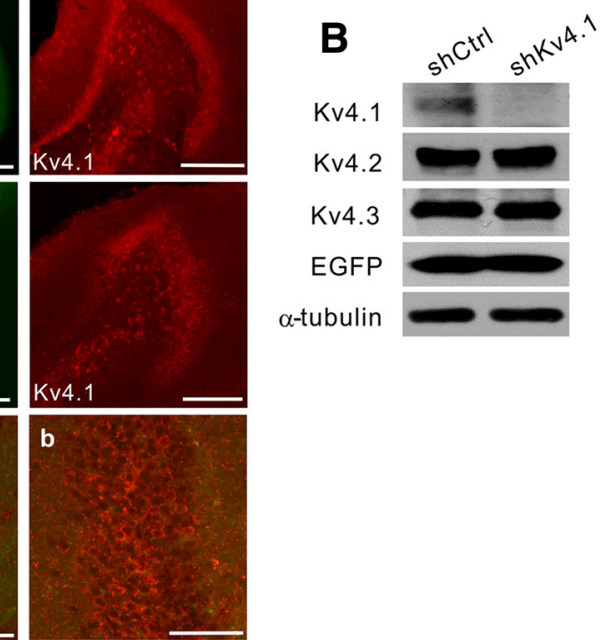

D

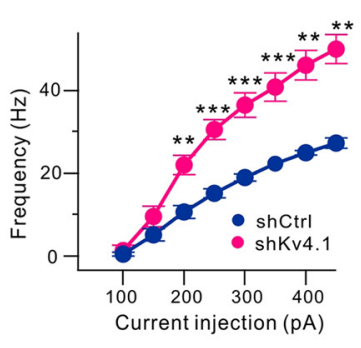

$\operatorname{RMP}(m V) \quad R_{\text {in }}(M \Omega)$

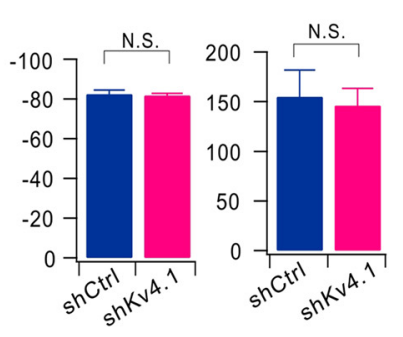

Figure 2. Knock-down of Kv4.1 increases firing frequency in GCs. A, Hippocampal sections from mice infected with AAV-shCtrl (top) or AAV-shKv4.1 (middle) were coimmunostained with anti-Kv4.1 and anti-GFP antibodies. DAPI was used to visualize cells in the hippocampus. (bottom) Merged image of both GFP (green) and Kv4.1 (red) fluorescence shows the selective reduction of Kv4.1 signal in GCs expressing GFP (left) by shKv4.1 infection. Scale bar, $200 \mu \mathrm{m}$. High-magnification images of the area corresponding to the boxes (a, b) shown in left panels (middle, right). Scale bar, $50 \mu \mathrm{m}$. B, Specificity and efficiency of Kv4.1 knock-down in cultured hippocampal neurons. Cultured hippocampal neurons were infected by AAV-shCtrl or AAV-shKv4.1 at DIV8 for $7 \mathrm{~d}$. At DIV15, endogenous expression levels of Kv4.1 were analyzed by Western blotting. $\mathbf{C a}$, Representative traces of the AP train recorded from the GCs in brain slices from shCtrl- and shKv4.1-injected mice in response to 150,200 and $250 \mathrm{pA}$ current injection (1 s duration). Cb. Averaged F-I curve in GCsinfected with AAV-shCtrl (dark blue, $n=11$ ) or AAV-shKv4.1 (pink, $n=9)$, ${ }^{* *} p<0.01$, ${ }^{* * *} p<0.001$ by Student's t-test. D, Knock-down of Kv4.1 had no effect on both RMP and $R_{\text {in }}$ in GCs. Bar graphs represent RMP (left) and $R_{\text {in }}$ (right) measured from GCs infected with AAV-shCtrl ( $n=11$ ) or AAV-shKv4.1 $(n=9)$. N.S., Not significant, $p>0.05$ (Student's t-test).

(Fig. $4 A, B$ ), confirming the specificity of the Kv4.1 antibody for blocking Kv4.1 currents. We then examined the effects of Kv4.1 inhibition in LF-GCs using intracelluar perfusion $(0.3 \sim 1 \mu \mathrm{g} / \mathrm{ml})$ of the Kv4.1 antibody (Fig. 4C). After patch break-in, AP firing in response to $200 \mathrm{pA}$ current injection increased gradually (filled circles, Fig. 4C) in association with a shortening of the first AP onset time (open circles, Fig. 4C). The mean firing frequency and the first AP onset time obtained after changes reached steady state $(17.0 \pm 2.4 \mathrm{~Hz}$ and $0.063 \pm 0.008 \mathrm{~s}, n=6)$ were significantly different from those obtained from the responses to the first stimulus $(8.6 \pm 0.8 \mathrm{~Hz}$ and $0.122 \pm 0.008 \mathrm{~s} ; n=6)$, whereas RMP was not significantly changed (open squares, Fig. $4 C$; from $-75.8 \pm$ $1.0 \mathrm{mV}$ to $-76.7 \pm 1.0 \mathrm{mV}, n=6, p=0.57$ ). There was no significant difference between the mean $R_{\text {in }}$ value measured in the presence of Kv4.1 antibody (150.9 $\pm 7.6 \mathrm{M} \Omega, n=23)$ and the value obtained from LF-GCs under control conditions (153.6 \pm $6.6 \mathrm{M} \Omega, n=20)(p=0.68$, Fig. $4 D)$. Effect of Kv4.1 inhibition in LF-GCs was also confirmed by comparing $F-I$ curves obtained from Kv4.1 antibody-perfused LF-GCs (red circles, Fig. 4E) with those from control LF-GCs shown in Figure $3 E$ (blue circles, Fig. $4 E)$. There was no significant shift in threshold current, but the slope increased markedly in Kv4.1 antibody-perfused GCs com- pared with the slope in control LF-GCs (Fig. 4E). I-O gain obtained between 100 and $200 \mathrm{pA}$ input current in the presence of Kv4.1 antibody $(19.6 \pm 1.9, n=10)$ was significantly higher than that in control LF-GCs $(8.6 \pm 1.1, n=12, p<0.001$, Fig. $4 F)$ or in shCtrl-infected LF-GCs, and not significantly different from the value in shKv4.1-infected GCs ( $p=0.81$, Fig. $4 F)$ or $I-O$ gain between 0 and $100 \mathrm{pA}$ input current in HF-GCs $(23.9 \pm 2.2, n=$ $13, p=0.16$, Fig. $4 F)$.

To further understand the role of Kv4.1 in firing frequency regulation in GCs, we pooled all data obtained under different experimental conditions and plotted the firing frequencies of individual GCs induced by $200 \mathrm{pA}$ injection against their $R_{\mathrm{in}}$. Data obtained from HF-GCs under control conditions (black circles, Fig. $4 G$ ) together with data obtained under Kv4.1-inhibited conditions with shKv4.1 or Kv4.1 antibody (red circles, Fig. $4 G$ ) represent the relationship between $R_{\mathrm{in}}$ and firing frequency when the contribution of Kv4.1 current is negligible, showing that firing frequency decreases with the decrease of $R_{\text {in }}$. However, data points for LF-GCs under control condition (blue circles, Fig. 4G) were below this relationship, suggesting that the low frequency of LF-GCs cannot be fully explained by low $R_{\text {in }}$, but Kv4.1 provides an additional outward shunting conductance upon depolar- 

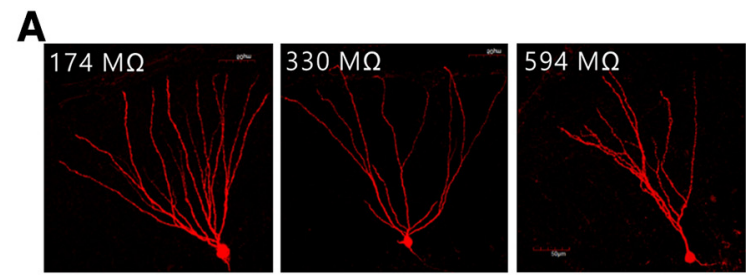

\section{$\mathrm{Ca}$}
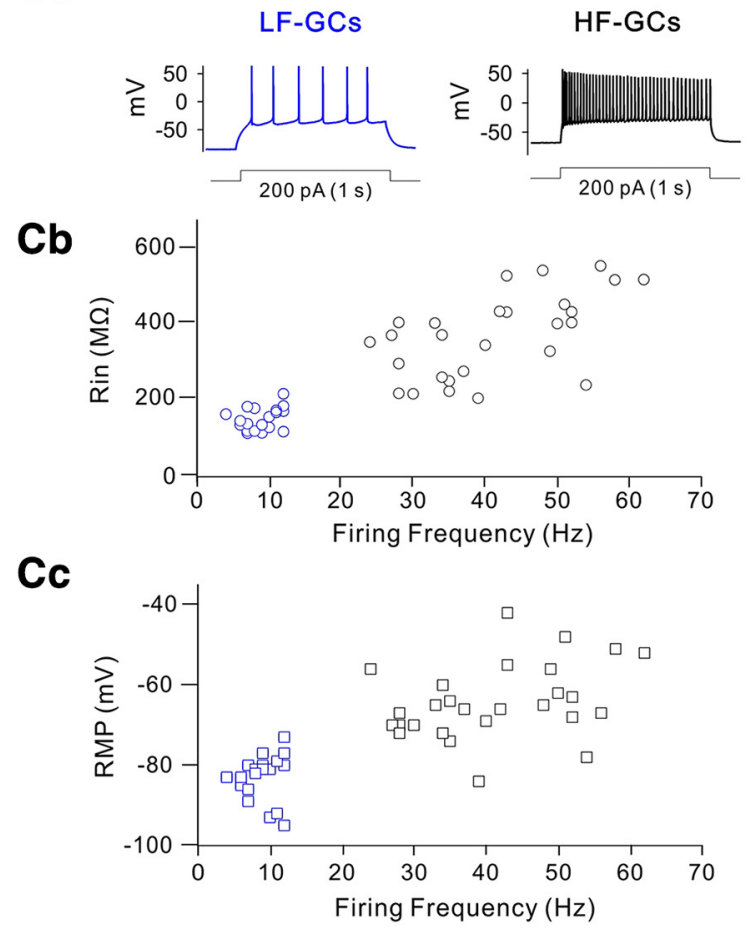
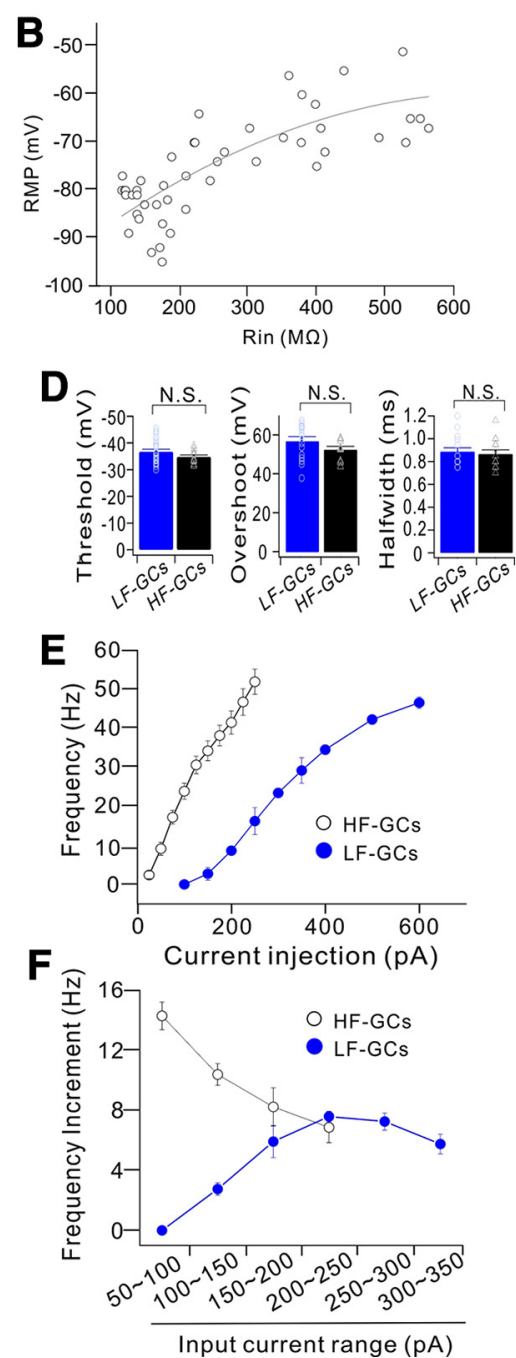

Figure 3. GCs cluster into two distinct populations based on the firing frequency. $A$, Sample images of GCs with different $R_{\text {in }}$ values. $B$, Plots of RMP values against $R_{\text {in. }}$ Data obtained from 47 cells are fitted to a polynomial function: $\mathrm{RMP}(\mathrm{mV})=-97.6+0.11 R_{\text {in }}+0.000087 R_{\text {in }}{ }^{2}$. C, Firing frequency of the repetitive APs evoked by a depolarizing current injection showed two distinct groups. Representative traces for APs of low-frequency (LF)- and high-frequency (HF)-GCs in response to $200 \mathrm{pA}$ (1 s duration) current injection (Ca). Plots for $R_{\text {in }}(\boldsymbol{C b})$ and RMP (Cc) against firing frequency at $200 \mathrm{pA}$ showed that firing frequency decreased with a decrease in $R_{\text {in }}$ and RMP, reaching the lowest level at $R_{\text {in }}<200 \mathrm{M} \Omega$ and at RMP below $-75 \mathrm{mV}$. D, No significant difference in AP threshold $(p=0.16)$, overshoot $(p=0.13)$, and AP width $(p=0.64)$ were found between LF-GCs and HF-GCS, N.S. (not significant) $p>0.05 . E$, The firing frequency plotted against the injected currents ( $F$-I curve) in LF- (filled blue circle, $n=12$ ) and HF- (open black circle, $n=13$ ) GCs. HF-GCs fired more action potentials in response to smaller current injection than LF-GCs. $F$, Increment in firing frequency for given increases in input currents in LF-GCs (filled blue circle) and HF-GCs (open black circle).

ization to further lower firing frequency. In contrast, the firing frequencies of HF-GCs, CA1-PCs and Vgat-expressing interneurons in DG were unaffected by Kv4.1 antibody (Fig. 4H-J), which is consistent with the weak expression of Kv4.1 in CA1-PCs (Fig. 1) and DG interneurons (Fig. 4Jc).

These results suggest that Kv4.1 selectively contributes to frequency regulation in LF-GCs.

\section{Distinct biophysical properties of Kv4.1 currents underlie its function as a frequency regulator}

The selective role of Kv4.1 in LF-GCs suggests a higher amplitude of Kv4.1 currents in LF-GCs. To verify this possibility, we tested the effects of Kv4.1 antibody on outward $\mathrm{K}^{+}$currents evoked by a depolarizing voltage step from a holding potential of $-70 \mathrm{mV}$ to $+30 \mathrm{mV}$ ( $1 \mathrm{~s}$ duration). In voltage-clamp experiments, we regarded GCs with $R_{\text {in }}$ smaller than $200 \mathrm{M} \Omega$ as LF-GCs, whereas GCs with $R_{\text {in }}$ ranging from 300 to $600 \mathrm{M} \Omega$ as HF-GCs, since LF-GCs and HF-GCs can be also distinguished with respective to $R_{\text {in }}$ value of $\sim 200 \mathrm{M} \Omega$ (Fig. $3 C b$ ). We monitored the changes in peak outward current amplitudes $\left(I_{\text {peak }}\right)$ during intracellular dialysis of GCs with internal solution containing Kv4.1 antibody $(0.3 \sim 1 \mu \mathrm{g} / \mathrm{ml})$, and found that $I_{\text {peak }}$ decreased gradually to a significant extent, reaching a steady state usually within $15 \mathrm{~min}$ in LF-GCs (Fig. $5 A, D$ ). In contrast, the effects of Kv4.1 antibody on $I_{\text {peak }}$ were negligible in HF-GCs (Fig. $5 A, D$ ), LF-GCs from shKv4.1 transfected mouse (Fig. 5B,D), and CA1-PCs (Fig. $5 C, D)$, where $\mathrm{Kv} 4.1$ had no significant role in regulating firing frequency (Fig. 4H,I). These results suggest that Kv4.1 significantly contributes to outward $\mathrm{K}^{+}$current only in LF-GCs, leading to the selective role of Kv4.1 in LF-GCs.

To further understand the mechanism underlying the role of Kv4.1 as a frequency regulator in LF-GCs, we analyzed the electrophysiological characteristics of Kv4.1 currents. To this end, we first analyzed the difference in outward $\mathrm{K}^{+}$currents between LF-GCs and HF-GCs. Whole-cell $\mathrm{K}^{+}$currents evoked by applying depolarization pulses in a $10 \mathrm{mV}$ step from the holding potnetial of $-70 \mathrm{mV}$ were dissected into TEA-sensitive currents $\left(\mathrm{I}_{\mathrm{TEA}}\right)$ and 4-AP-sensitive currents $\left(I_{4-\mathrm{AP}}\right)$ using $3 \mathrm{mM}$ TEA and 5 
A
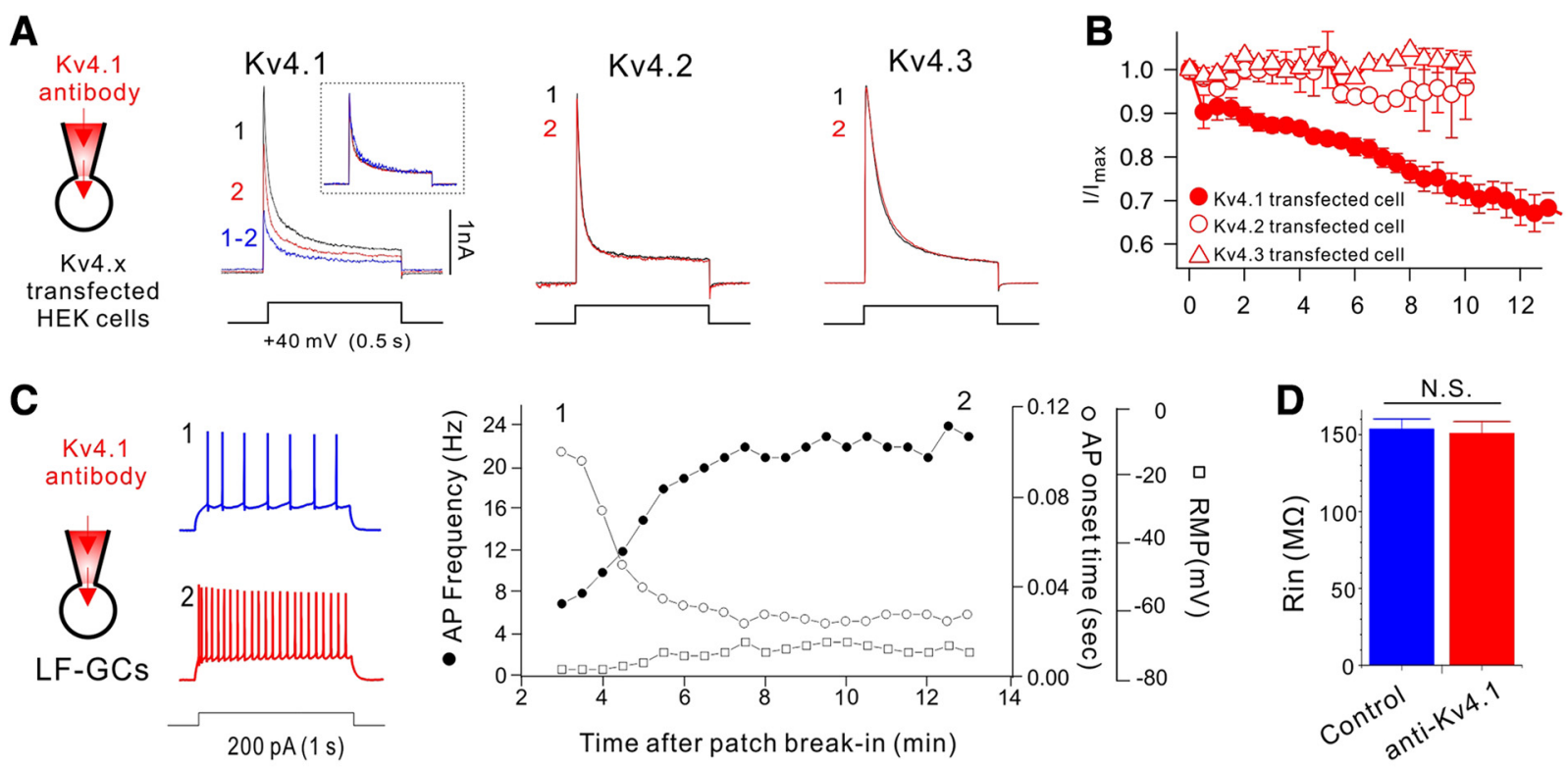
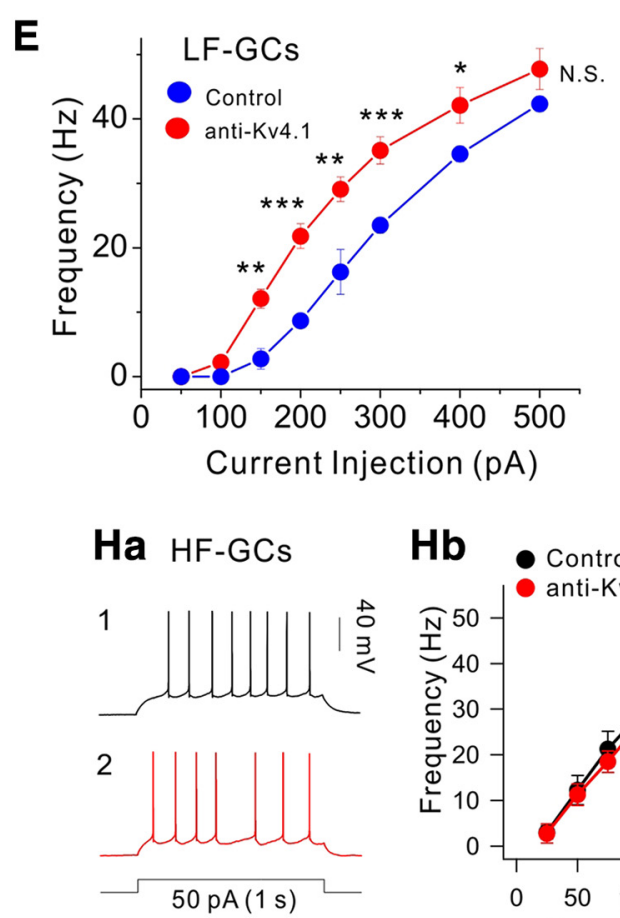

Ja
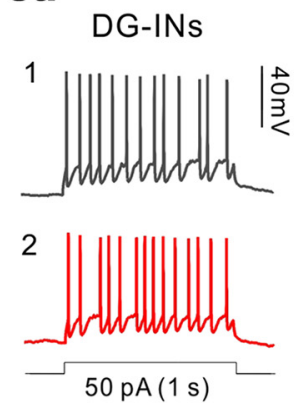

Jb

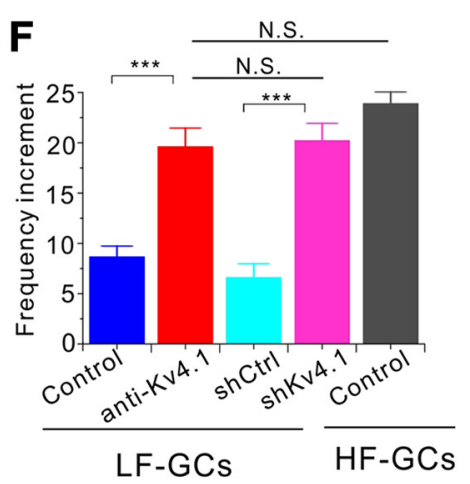

la CA1-PCs
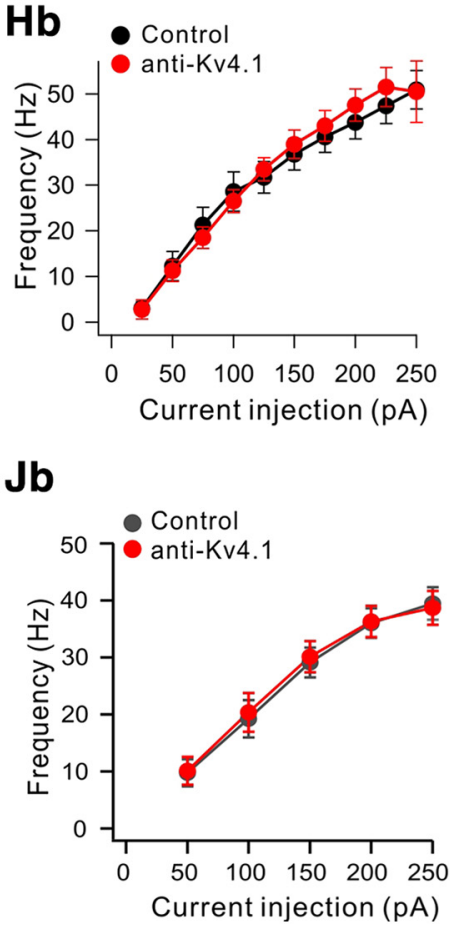

Jc

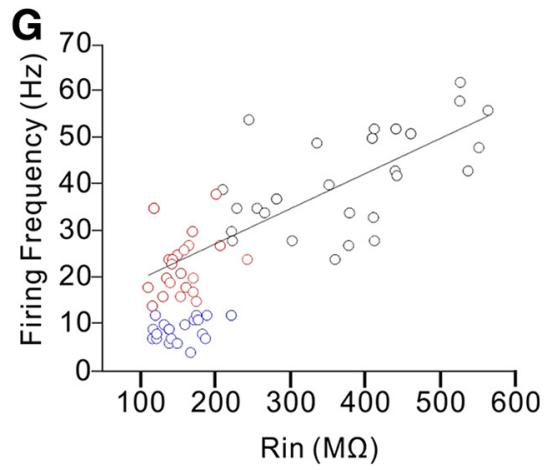

Ib

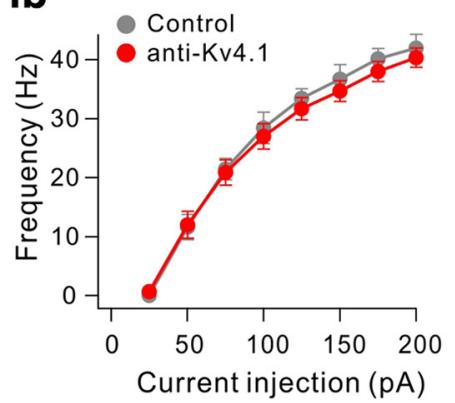

Kv4.1
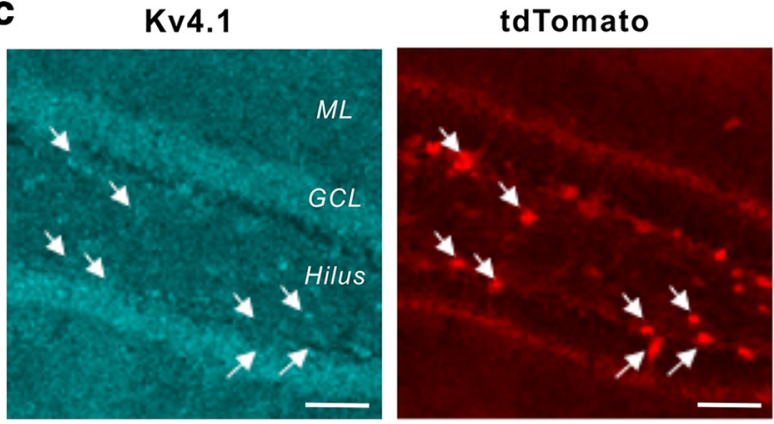

Figure 4. Kv4.1 inhibition increases firing frequency selectively in LF-GCS. A, The specificity of Kv4.1 antibody for the Kv4.1-mediated current was determined in HEK293 cells expressing Kv4.1, Kv4.2 or Kv4.3. Outward currents evoked by depolarizing pulse to $+40 \mathrm{mV}$ for $1 \mathrm{~s}$ in Kv4.1, Kv4.2 or Kv4.3-expressing HEK293 cells measured just after patch break-in (1, black) and >12 min after intracellular application of the Kv4.1 antibody (2, red). Inset, $\mathrm{K}^{+}$currents measured before (black) and after (red) inhibition of Kv4.1 and subtracted current (blue) in (Figure legend continues.) 
mM 4-AP, respectively. Average current traces ( $n=9$ for LF-GCs, $n=4$ for HF-GCs) showed that the amplitude of $\mathrm{I}_{\mathrm{TEA}}$ was larger in LF-GCs compared with HF-GCs (Fig. 6A), but there was no significant difference when the amplitude was normalized to the cell capacitance (Fig. $6 \mathrm{~B}$ ), indicating that the density of $\mathrm{I}_{\mathrm{TEA}}$ was not significantly changed with the decrease in $R_{\text {in }}$ in this range. With respect to $I_{4-\mathrm{AP}}$, not only the current amplitude (Fig. 6C) but also the current density normalized to the cell capacitance (Fig. 6Da) was significantly larger in LF-GCs compared with those of HF-GCs. Furthermore, there was a distinctive feature in $I_{4-\mathrm{AP}}$ between LF- and HF-GCs. $I_{4-\mathrm{AP}}$ in HF-GCs showed rapidly inactivating kinetics and the current amplitude measure at the end of the pulse $\left(I_{\mathrm{ss}}\right)$ was negligible, whereas a considerable proportion of $I_{4-\mathrm{AP}}$ in LF-GCs was not fully inactivated even after $1 \mathrm{~s}$ $\left(I_{\text {ss }} / I_{\text {peak }}\right.$ at $+30 \mathrm{mV}, 0.24 \pm 0.02, n=9$, Fig. $\left.6 \mathrm{Db}\right)$. The difference in $I_{4-\mathrm{AP}}$ between LF- and HF-GCs may be attributable to the contribution of Kv4.1 currents in LF-GCs.

Kv4.1 currents are known to have similar electrophysiological characteristics with Kv4.2 or Kv4.3 currents when studied in heterologous expression systems (Nakamura et al., 2001), but its kinetics in native neurons is unclear. Different kinetics of $I_{4-\mathrm{AP}}$ in LF- and HF-GCs suggests that Kv4.1 current may have different kinetics from typical A-type $\mathrm{K}^{+}$currents $\left(I_{\mathrm{A}}\right)$. We obtained Kv4.1 currents $\left(I_{\mathrm{Kv} 4.1}\right)$ from LF-GCs in the presence of $3 \mathrm{~mm}$ TEA to inhibit delayed rectifier $\mathrm{K}^{+}$currents by subtracting the currents measured 15 min after patch break-in in the presence of Kv4.1 antibody from currents recorded immediately after break-in (Fig. $6 E$ ). Subtraction results showed that $I_{\mathrm{Kv} 4.1}$ inactivated slowly, and thus a significant proportion of currents remained at the end of

\footnotetext{
$\leftarrow$

(Figure legend continued.) the Kv4.1-expressing cell were normalized to the peak amplitude of current. $\boldsymbol{B}$, Summary graph of inhibition of $\mathrm{I} / \mathrm{I}_{\max }$ by Kv4.1 antibody in HEK293 cells expressing Kv4.1 (closed circle), Kv4.2 (open circle), or Kv4.3 (open triangles). Values indicate mean \pm SEM. Error bars indicate SEM. $\boldsymbol{C}-\boldsymbol{H}$, Effects of Kv4.1 antibody on intrinsic excitability in LF-GCS $(\boldsymbol{C}-\boldsymbol{G})$ and HF-GCs $(\boldsymbol{H})$. $\boldsymbol{C}$, Example traces (left) of APs at the time points $(1,2)$ indicated on the right graph. Right, Graphs showing the time course of the alterations in RMP (open squares), firing frequency (filled circles) and first AP onset time to $1 \mathrm{~s}$ current injection (open circles) during Kv4.1 antibody perfusion in LF-GCS. $D$, Mean values for $R_{\text {in }}(n=6)$ measured within 3 min after patch break-in and after the changes in firing frequency induced by Kv4.1 antibody reached steady state (12 min). $\boldsymbol{E}$, The $F-I$ curve measured from LF-GCs in the presence of Kv4.1 antibody in the pipette solution (red closed circles, $n=10$ ). The same data shown in Figure $3 E$ (blue closed circles, LF-GCS) are superimposed to compare with the $F-I$ curve obtained in the presence of Kv4.1 antibody. Significance levels for the difference between LF-GCS and antiKv4.1 were indicated. $P$ values at $150,200,250,300,400$, and 500 pA were $0.0024,<0.0001$, $0.0048,<0.0001,0.0035$, and 0.189 , respectively. $F, I-0$ gain between 100 and $200 \mathrm{pA}$ input currents obtained from each condition. Increases in $1-0$ gain by Kv4.1 inhibition were significant $(p<0.001){ }^{*} p<0.05,{ }^{* *} p<0.01,{ }^{* * *} p<0.001$, N.S. (not significant) $p>0.05$ by Student'st-test. G, Firing frequency of GCs under control condition (blue circles for LF-GCs; black circles for HF-GCS) and Kv4.1-inhibited conditions using shKv4.1 or Kv4.1 antibody (red circles) was plotted against $R_{\text {in }}$. The firing frequency of HF-GCs (black circles) and Kv4.1-depleted LF-GCS (red circles) showed a linear relationship with $R_{\text {in, }}$, whereas the firing frequency of LF-GCS (blue circles) is lower. $\boldsymbol{H}$, The Kv4.1 antibody had no effects on intrinsic excitability in HF-GCs. $\mathbf{H a}$, Representative traces of AP trains obtained from $\mathrm{HF}-\mathrm{GCS}$ recorded right after patch break-in (1, black) and $15 \mathrm{~min}$ after intracellular application of Kv4.1 antibody (2, red). $\mathbf{H b}$, Inhibition of Kv4.1 had no effect on the firing frequency of HF-GCS $(n=4)$. Ia, Representative traces from CA1-PCs recorded right after patch break-in (1, gray) and 15 min after intracellular application of Kv4.1 antibody (2, red). $\mathbf{l b}$, Firing frequency was plotted against the injecting currents (1 s) with or without Kv4.1 antibody in the pipette for CA1-PCs $(n=7)$. Ja, Representative traces of AP trains in DG interneurons from Vgat-ires-Cre mice infected with AAV-DI0-mCherry recorded at 3 (1, black trace) and 13 (2, red trace) min after patch break-in. Jb, Relationships between the firing frequency and the current injected were not changed by Kv4.1 antibody.Jc, Hippocampal section from Vgat-tdTomato mice was coimmunostained with Kv4.1 and tdTomato antibodies. Arrows indicates Vgat-positive interneurons expressing tdTomato. Scale bar, $50 \mu \mathrm{m}$.
}

the $1 \mathrm{~s}$ depolarization (Fig. 6E). When $5 \mathrm{~mm} 4$-AP was applied after $I_{\mathrm{Kv} 4.1}$ was completely inhibited by the Kv4.1 antibody, there was further reduction in outward currents (Fig. 6F). The 4-APsensitive currents obtained in the presence of Kv4.1 antibody by subtraction, which were referred to as $I_{4-\mathrm{AP}-\mathrm{Kv} 4.1}$ (Fig. $6 F$ ), showed a rapid voltage-dependent activation followed by rapid inactivation, the characteristics of which are consistent with the kinetics known for classical A-type $\mathrm{K}^{+}$currents. Current-voltage relationships for $I_{\mathrm{Kv} 4.1}$ and those for $I_{4-\mathrm{AP}-\mathrm{Kv} 4.1}$ measured at its peak and steady-state ( $1 \mathrm{~s})$ showed a clear difference in steadystate currents (Fig. 6Eb, Fb). To compare the difference between inactivation kinetics of $I_{\mathrm{Kv} 4.1}$ and $I_{4-\mathrm{AP}-\mathrm{Kv} 4.1}$ more clearly, averaged trace for $I_{\mathrm{Kv} 4.1}$ (obtained from 7 cells) and that for $I_{4-\mathrm{AP}-\mathrm{Kv} 4.1}$ (obtained from 10 cells) obtained at $+30 \mathrm{mV}$ were superimposed, showing that inactivation kinetics for $I_{\mathrm{Kv} 4.1}$ was distinctively slower than that for $I_{4-\mathrm{AP}-\mathrm{Kv} 4.1}$ (Fig. $6 G a$ ). As a parameter for inactivation kinetics, the ratio of $I_{\text {ss }}$ to $I_{\text {peak }}\left(I_{\mathrm{ss}} / I_{\text {peak }}\right)$ was obtained, showing that the value for $I_{\mathrm{Kv} 4.1}$ was significantly larger than that for $I_{4-\mathrm{AP}-\mathrm{Kv} 4.1}$ (Fig. $6 G b$ ). Intriguingly, $I_{\mathrm{ss}} / I_{\text {peak }}$ for $I_{\mathrm{Kv} 4.1}$ was not very different between -10 and $50 \mathrm{mV}$, suggesting that voltage dependence of $\mathrm{I}_{\mathrm{KV} 4.1}$ inactivation was not steep. In contrast, normalized conductance $\left(G / G_{\max }\right)$ of $I_{\mathrm{Kv} 4.1}$ and that of $I_{4-\mathrm{AP}-\mathrm{Kv} 4.1}$ were fitted well with Boltzmann equations with the voltage for half activation $\left(\mathrm{V}_{1 / 2}\right)$ of $7.58 \pm 2.24 \mathrm{mV}(n=5)$ for $I_{\mathrm{Kv} 4.1}$ and $-0.61 \pm 2.63 \mathrm{mV}(n=6)$ for $I_{4-\mathrm{AP}-\mathrm{Kv} 4.1}$, indicating voltage dependence of activation for both $I_{\mathrm{Kv} 4.1}$ and $I_{4-\mathrm{AP}-\mathrm{Kv} 4.1}$ (Fig. 6H). Together, these results suggest that large sustained currents for $I_{4-\mathrm{AP}}$ in LF-GCs (Fig. $6 \mathrm{C}$ ) are attributable to slowly inactivating $I_{\mathrm{Kv} 4.1}$ and that sustained outward currents due to slow inactivation kinetics may support the roles of Kv4.1 as a frequency regulator during repetitive activity.

Since the slow inactivation kinetics of $I_{\mathrm{Kv} 4.1}$ in GCs resemble D-type $\mathrm{K}^{+}$current $\left(I_{\mathrm{D}}\right)$ (Storm, 1988; Beck et al., 1997), which shows high sensitivity to 4-AP, we examined 4-AP sensitivity of Kv4.1 currents. To this end, the effect of Kv4.1 antibody on outward $\mathrm{K}^{+}$currents was tested in the presence of a low and a high concentration $(40 \mu \mathrm{M}$ and $5 \mathrm{~mm})$ of 4-AP. Kv4.1 antibody reduced outward currents in the presence of $40 \mu \mathrm{M}$, but not $5 \mathrm{~mm} 4$-AP (Fig. $6 I a, I b$ ). The fact that a high concentration of 4-AP is required to inhibit $I_{\mathrm{Kv} 4.1}$ suggests that $I_{\mathrm{Kv} 4.1}$ is distinctive from $I_{\mathrm{D}}$.

\section{Kv4.1 expression is developmentally regulated}

$R_{\text {in }}$ is inversely correlated with dendritic length, which increases with maturation (Dieni et al., 2013). Contribution of Kv4.1 currents to frequency regulation exclusively in LF-GCs where $R_{\text {in }}$ is mostly lower than $200 \mathrm{M} \Omega$ may suggest that Kv4.1 expression is developmentally regulated. To investigate this possibility, we examined Kv4.1 expression in the DG of 3-week-old mice and found that Kv4.1 signal was much weaker, in inner GCL than outer GCL, with cells located at the hilar border showing lower expression (Fig. 7A). When newly generated neurons were identified with the doublecortin (DCX) antibody, Kv4.1 signals were hardly found in $\mathrm{DCX}^{+}$cells located in the deep layer (Fig. 7A), supporting the maturation-dependent change in Kv4.1 expression. In contrast to Kv4.1, Kv4.2 signals were detected in both DCX-positive and mature GCs in the DG (Fig. 7B). Consistent with this observation, the protein levels of Kv4.1 but not Kv4.2 was higher in DG lysates from 3-week-old mice than those from 8-week-old mice (Fig. 7C). 
A

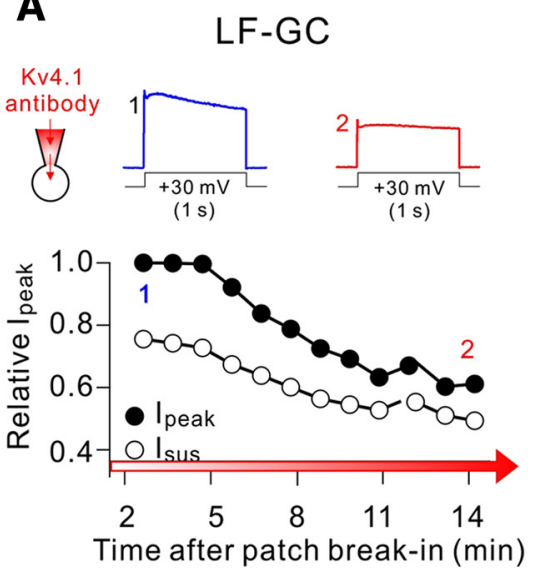

HF-GC
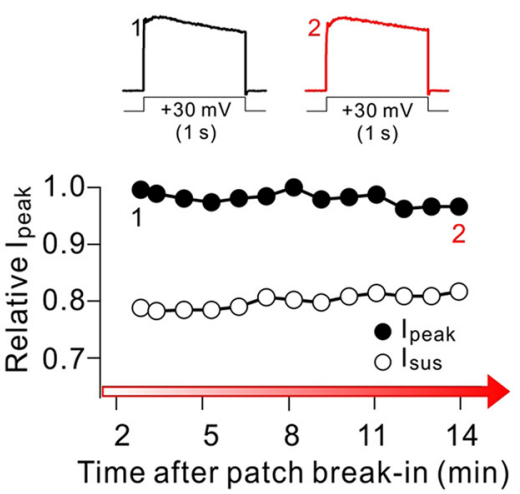

B

\section{shKv4.1/LF-GCs}
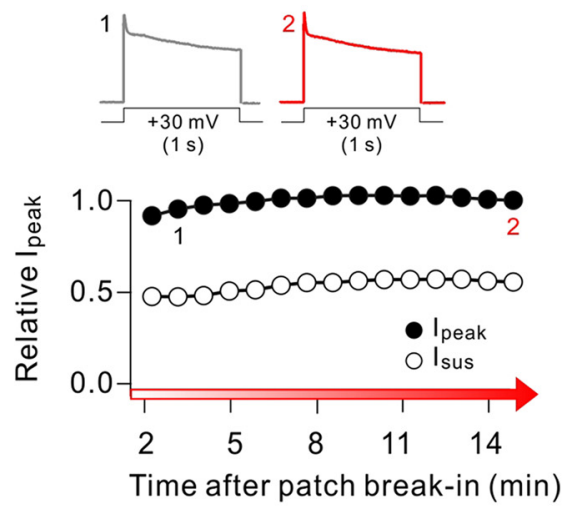
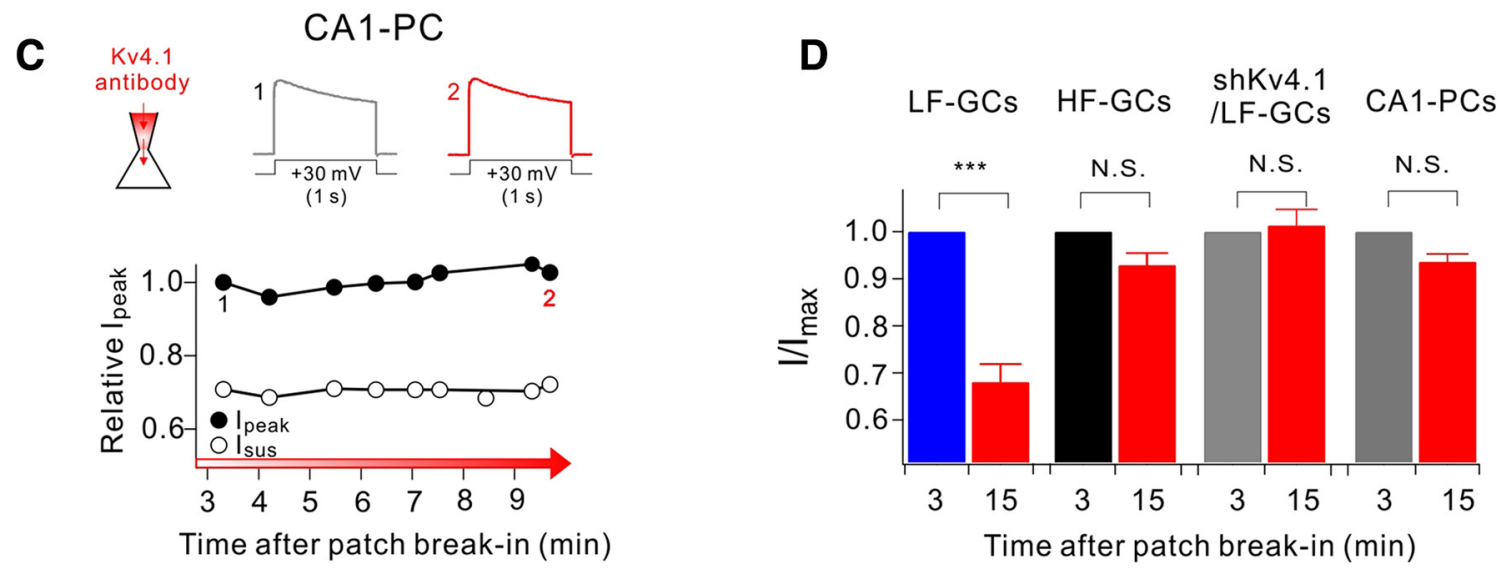

Figure 5. Kv4.1 inhibition reduces $\mathrm{K}^{+}$current selectively in LF-GCs. $\boldsymbol{A}-\boldsymbol{C}$, Effects of Kv4.1 antibody on outward K ${ }^{+}$currents in LF- and HF-GCS (A), LF-GCs infected by shKv4.1 (B), and CA1-PCS (C). (top) Representative current traces recorded shortly after patch break-in usually within 3 min (marked with 1) and those recorded after $10-15$ min (marked with 2). A significant decrease in outward $\mathrm{K}^{+}$currents by Kv4.1 antibody was observed only in LF-GCs. Voltage protocol was depicted below current traces. (bottom) The current amplitude measured at the peak (I to the maximum amplitude during recording period were plotted against the time after patch break-in. $D, I_{\text {peak }}$ obtained at 15 min after patch break-in normalized to that obtained at 3 min after patch break-in were pooled and plotted as bars with SEM. LF-GCs $(n=15)$, HF-GCs $(n=8)$, shKv4.1-infected LF-GCs $(n=7)$, CA1-PCs $(n=9)$. Paired $t$ test. ${ }^{* *} p<0.001 .$, N.S. (not significant) $p>0.05$.

\section{Impaired pattern separation in mice depleted of $\mathrm{Kv} 4.1$} transcripts in DG

Sparse activity is one of the key features that enable the DG to function as a pattern-separator (Treves and Rolls, 1994). To investigate the possibility that Kv4.1 currents in LF-GCs contribute to sparse activity of DG networks and thus to pattern separation, we used a contextual fear conditioning paradigm (McHugh et al., 2007) and tested whether mice with their Kv4.1 transcripts knocked down in GCs exhibit an impairment in discriminating similar contexts. We confirmed that 3 weeks after the injection of shKv4.1, the expression of GFP was localized in the GCL in the DG and mossy fiber (MF) (Fig. 8A). We first examined the locomotor activity and the anxiety levels of mice using the open field test and elevated plus maze test. Both the shCtrl-injected controls and shKv4.1-injected mice showed similar exploratory patterns as they moved the same distance in the open filed box, and they spent a similar amount of time in the center zone (Fig. $8 B$ ). In addition, both groups spent the same amount of time in the open and closed arms of elevated plus maze (Fig. 8C).

For behavioral pattern separation analysis, shCtrl-injected controls and shKv4.1-injected mice were tested in a contextual fear conditioning task for the first three days using a context (referred to as 'context A') in which animals received a single foot shock 3 min after being introduced to the context (Fig. 8D). On days 4 and 5, we prepared another context similar to the context A and named it 'context B'. context B was composed of an identical metal grid floor but had an odor ( $1 \%$ acetic acid), dimmer lighting ( $50 \%$ of context $\mathrm{A}$ ), and a sloped floor ( $15^{\circ}$ angle). Mice in control and shKv4.1 groups were each divided into two subgroups. One subgroup visited context A on day 4 and context B on day 5 , and the other subgroup visited context B on day 4 and context A on day 5. Neither group received a foot shock in either context on days 4 and 5 and freezing was evaluated for $5 \mathrm{~min}$. On days 4 and 5, both the control and shKv4.1-injected groups showed similar freezing levels during the 5 min test in context A (Fig. 8Ea) and appeared to generalize between the two similar contexts (Fig. 8Eb, two-way ANOVA, group, $F_{(1,32)}=0.46, p=$ 0.50 , context: $F_{(1,32)}=0.01, p=0.93$, group $\times$ context: $F_{(1,36)}=$ $0.00, p=1.00)$.

The mice were subsequently trained to discriminate between the two contexts by visiting both contexts daily for $7 \mathrm{~d}$ with a $2 \mathrm{~h}$ interval between the contexts (from day 6 to 12), always receiving a foot shock 3 min after being placed in context A but not in context $\mathrm{B}$. Daily discrimination ratio was calculated by determining the ratio of freezing during the 3 min period in context $A$ to total freezing during the two visits (A and B). On day 6, both 
A

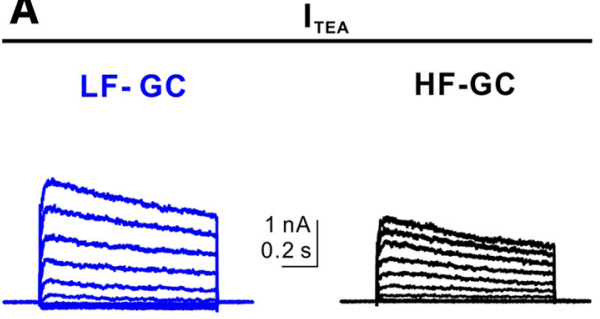

B

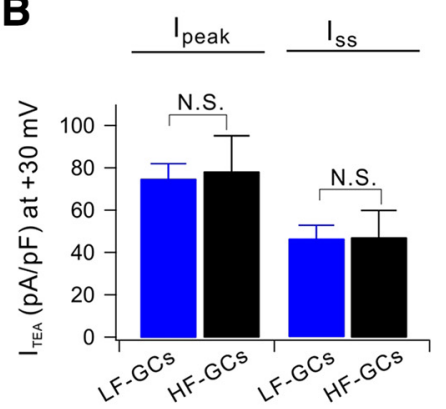

C $\quad \mathrm{I}_{4 \cdot \mathrm{AP}}$

LF-GC HF-GC

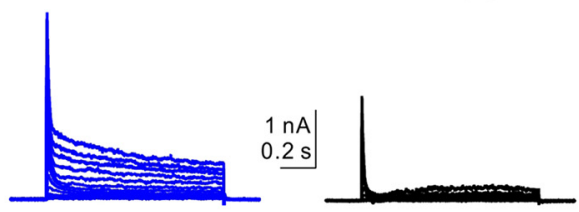

Db

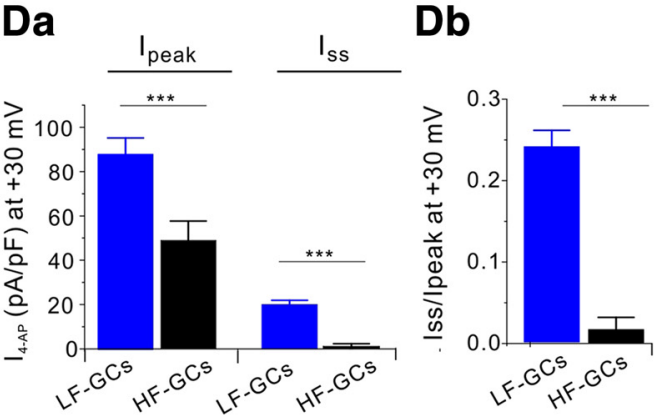

Ea

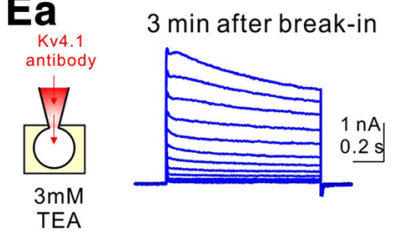

$\mathrm{Fa}$

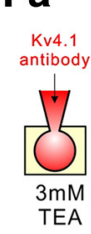

Ga

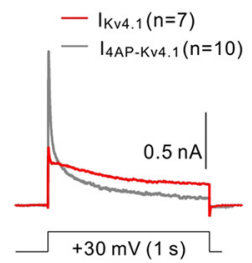

la

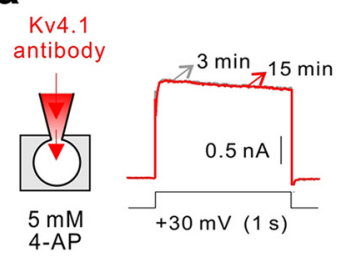

15 min after break-in

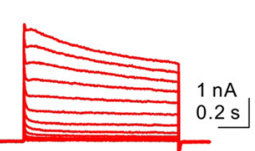

15 min after break-in
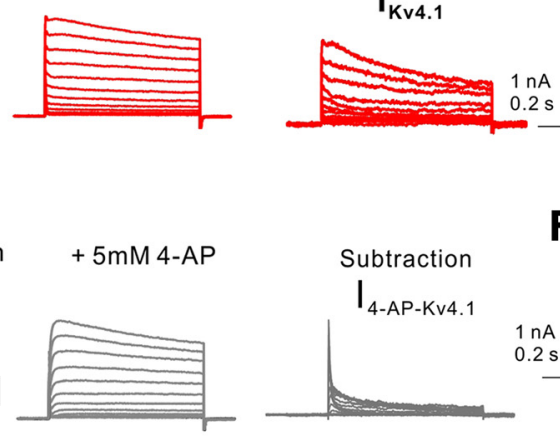

Fb

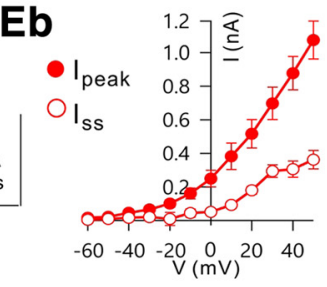

$-60-40-20 \underset{(\mathrm{mV})}{0} 20 \quad 40$
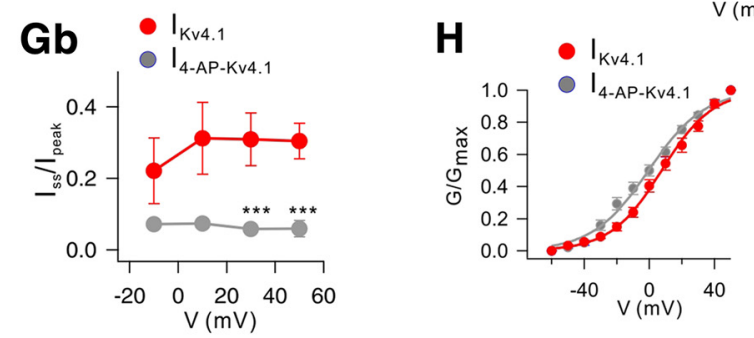

Kv4.1

antibody
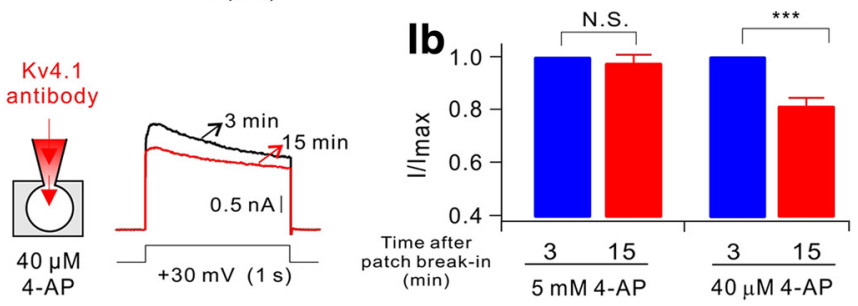

Figure 6. Kv4.1 contributes to slowly inactivating sustained outward currents in GCS. A, Average current traces for TEA-sensitive currents (l IFEA $\left._{1}\right)$ in LF-GCS (blue, $\left.n=9\right)$ and HF-GCS (black, $\left.n=4\right)$. $B$, Current density (pA/pF) of $I_{\text {peak }}$ and $I_{\text {sus }}$ at $+30 \mathrm{mV}$ for $\mathrm{I}_{\text {TEA }}$ was not different in LF-GCS (blue) and HF-GCs (black). C, Average current traces for 4-AP-sensitive currents ( $I_{4-\mathrm{AP}}$ ) in LF-GCS (blue, $n=$ 9) and HF-GCs (black, $n=4)$. Da $I_{4-A P}$ density in LF-GCs was significantly larger than that in HF-GCs $\left(I_{\text {peak }} p=0.001 ; I_{\text {sus }}, p=0.0001\right) ;$ Student'st-test. Db, The ratio of $I_{\text {ss }}$ to $I_{\text {peak }}\left(I_{\text {ss }} / I_{\text {peak }}\right)$ for $I_{\text {Kv4.1 }}$ was significantly enhanced in LF-GCS $(p=0.0005)$. $E$, Whole-cell, voltage-gated $\mathrm{K}^{+}$currents in LF-GCs at the beginning of the recording (blue) and after 15 min dialysis of Kv4.1 antibody (middle, red). The difference traces (right, red) represent currents mediated by Kv4.1 channel. Eb, Averaged $I-V$ relationships of difference traces at its peak $\left(I_{\text {peak }}\right)$ and at the end of the pulse $\left(I_{s s}\right)$ from experiments shown in $\mathbf{E a}$. Fa, Records showing subsequent effects of $5 \mathrm{~mm}$ 4-AP after the maximal effect of Kv4.1 antibody in LF-GCS. The difference traces (right, gray) are defined as $I_{4-A P-k v 4.1}$. Fb, Averaged $I-V$ relationships of $I_{4-\text { AP-Kv4.1 }}$ from experiments such as $F$ a show no sustained outward currents. (Ga) Averaged $I_{\text {Kv4.1 }}$ and $I_{4-A P-K v 4.1}$ from LF-GCs are superimposed. Gb, $I_{\text {sus }} / I_{\text {peak }}$ for $I_{\text {Kv4.1 }}$ (red) and $I_{4-A P-K v 4.1}$ (gray) of LF-GCS. H, Activation curve for $I_{\text {Kv4.1 }}$ (red) and $I_{\text {4-AP-Kv4.1 }}$ (gray) in LF-GCS. Ia, Impact of the Kv4.1 antibody internal on $\mathrm{K}^{+}$current during the bath application of 5 mM 4-AP and $40 \mu \mathrm{m} 4-\mathrm{AP}$. Ib, Summary bar graphs showing inhibition of $/ / I_{\max }$ by Kv4.1 antibody in the presence of $5 \mathrm{~mm} 4-\mathrm{AP}(n=4)$ or $40 \mu \mathrm{m} 4-\mathrm{AP}(n=4)$ in the bathing solution. N.S. (not significant) $p>0.05,{ }^{* * *} p<0.001$ by Student's t-test. Error bars indicate SEM. Values indicate mean \pm SEM. 
A
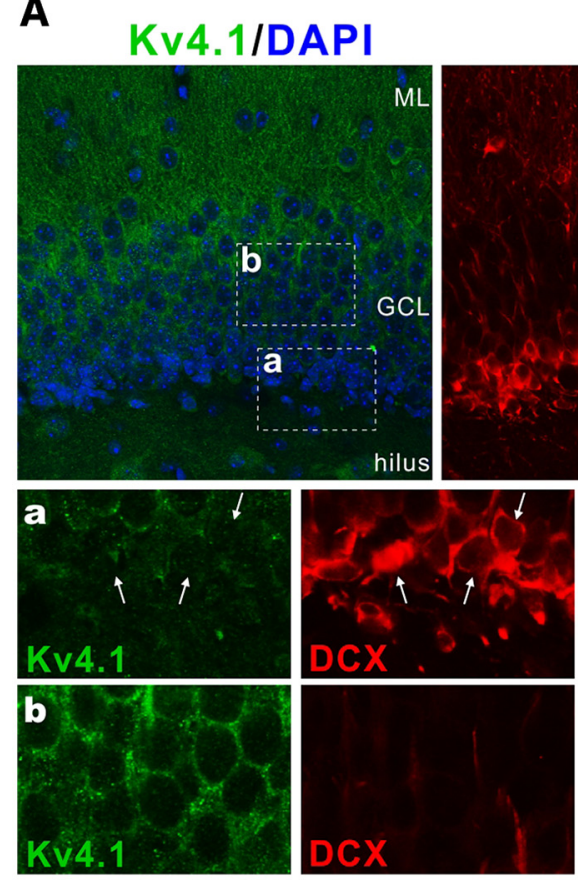

DCX
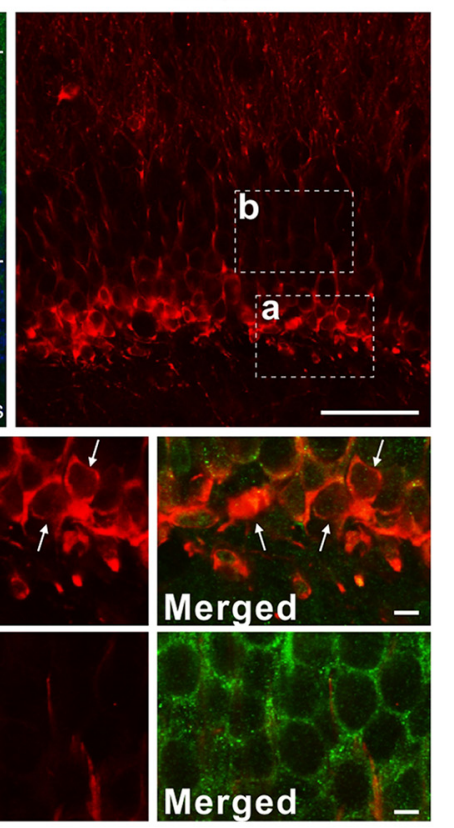

B
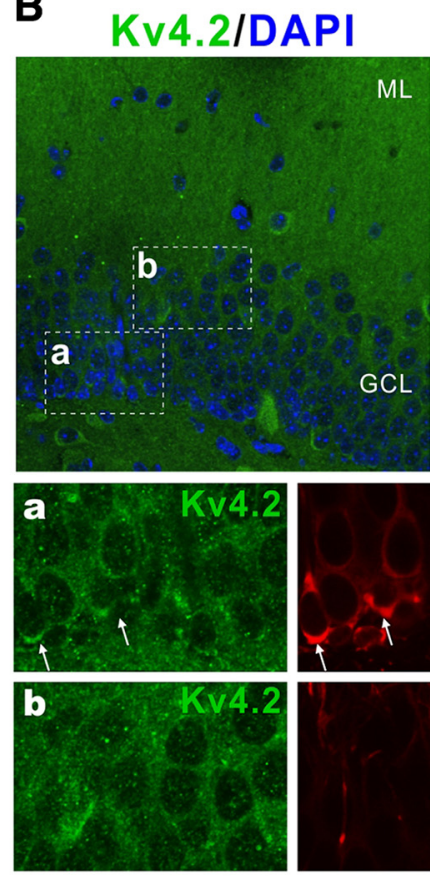
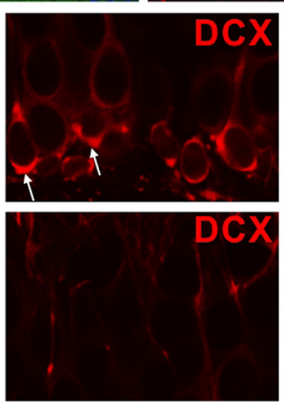

DCX
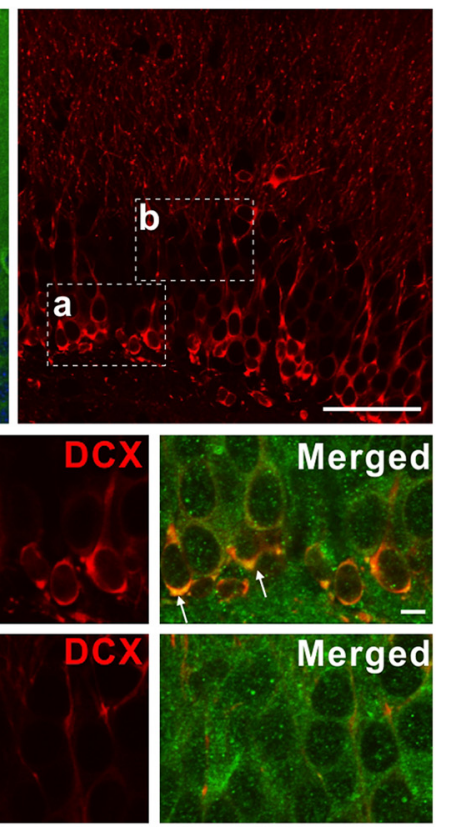
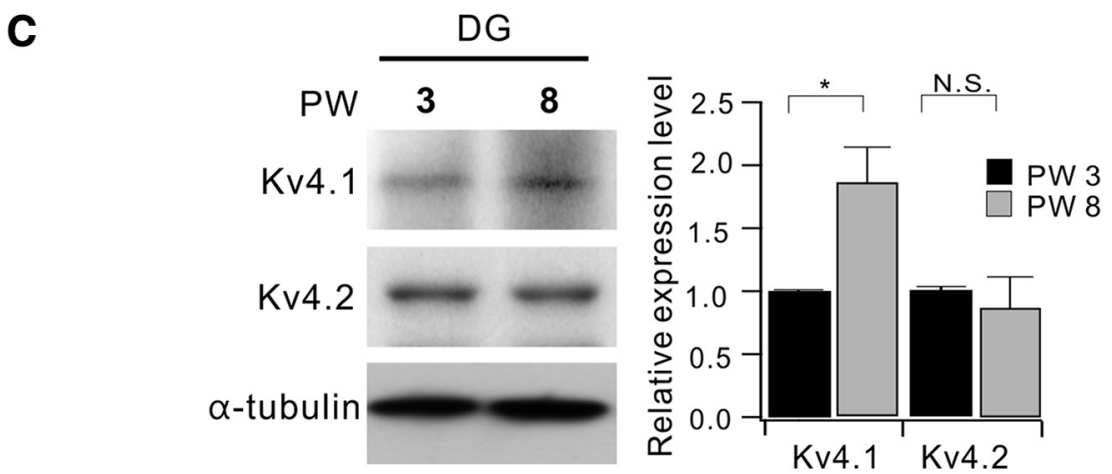

Figure 7. Expression levels of Kv4.1 in DG increase with development. $A$, Coimmunostaining of Kv4.1 (green) and doublecortin (DCX, red) in 3-week-old mice. Scale bar, $50 \mu \mathrm{m} . \boldsymbol{A}, \boldsymbol{B}$, Bottom, High-magnification images of the areas corresponding to the dashed box shown in top panels. Scale bar, $5 \mu \mathrm{m}$. B, Immunostaining of DG with Kv4.2 antibody (green) and DCX antibody (red) in 3-week-old mice. Scale bar, $50 \mu \mathrm{m}$. C, Western blot analysis for Kv4.1 and Kv4.2 in DG from 3- and 8-week-old mice. Bar graphs indicate the mean \pm SEM $(n=3)$. GCL, granule cell layer; ML, molecular layer. N.S. (not significant) $p>0.05,{ }^{*} p<0.05$ (Student's t-test).

groups did not distinguish between the contexts (Fig. $8 \mathrm{Fa}$; group, $F_{(1,32)}=0.13, p=0.72$; context, $F_{(1,32)}=0.21, p=0.64$, two-way ANOVA), and thus the discrimination ratio was $\sim 0.5$. As the experiment progressed, control mice began to discriminate context $\mathrm{B}$ from context $\mathrm{A}$, and the discrimination ratio increased accordingly. However, shKv4.1-injected mice exhibited high levels of freezing in the shock-free context until day 12 (Fig. $8 \mathrm{Fb}$, group: $F_{(1,16)}=54.55, p<0.001$, group $\times$ days: $F_{(6,96)}=2.49$, $p=0.028$, repeated measure two-way ANOVA). In Figure $8 F b$, the freezing levels on day 12 are compared between the two contexts for each group (control, $p=0.000002$; shKv4.1, $p=0.78$; paired $t$ test), showing deficits in the rapid acquisition of contextual discrimination.

We further examined the behavioral role of Kv4.1 in discrimination of similar objects using the novel object recognition test. During the familiarization phase, both control and shKv4.1injected mice were allowed to explore two identical sample objects for $10 \mathrm{~min}$. After a $24 \mathrm{~h}$ delay, one of the familiar objects was replaced by a similar novel object, and mice were tested for discriminating the novel object from a familiar one. The control group showed a capability of discriminating similar objects and explored the novel object longer (Fig. 8Ga). In contrast, shKv4.1injected mice spent a similar amount of time exploring familiar and novel objects. Consistent with a lack of preference for the novel object, discrimination index [(time spent exploring the novel object/total object exploration time) $\times 100 \%$ )] was significantly reduced in shKv4.1-injected mice (Fig. 8Gb). Collectively, these results suggest that the hyperexcitability of GCs induced by Kv4.1 knock-down specifically impairs pattern separation without affecting the locomotor activity or the general anxiety levels.

\section{Discussion}

In the present study, we identified Kv4.1 as a specific mechanism responsible for low-frequency firing of mature GCs. We found that subcellular distribution and biophysical properties of Kv4.1 are distinctive from those of Kv4.2 that mediates classical $I_{\mathrm{A}}$ (Figs. $1,6)$. We also showed that specific knock-down of Kv4.1 in the DG region selectively impaired conditional freezing between 
similar contexts, suggesting that lowfrequency firing of mature GCs is crucial for pattern separation.

\section{Changes in intrinsic excitability and firing behavior during maturation of GCs}

The DG is one of the regions where adult neurogenesis occurs. Changes in excitation/inhibition balance and intrinsic excitability are key components that occur during maturation of GCs. In contrast to the rigorous studies on maturationdependent synaptic changes (Marín-Burgin et al., 2012; Dieni et al., 2013), maturation-dependent changes in intrinsic excitability, in particular the mechanisms underlying firing frequency changes, are not fully understood. It is generally believed that hyperpolarization of RMP occurs in parallel with the decrease in $R_{\text {in }}$ during maturation, resulting in lowfrequency firing in mature GCs. Inwardly rectifying potassium channels $\left(\mathrm{K}_{\mathrm{ir}}\right)$ (Mongiat et al., 2009) and $\mathrm{GABA}_{\mathrm{B}}$ receptor-mediated GIRK currents (Gonzalez et al., 2018) were suggested to underlie maturation-dependent decrease in firing frequency in adult-born GCs. GIRK current appears in developing GCs with $R_{\text {in }}$ between 1 and $2 \mathrm{G} \Omega$, which corresponds to $\sim 3$ weeks of age (Gonzalez et al., 2018), whereas $\mathrm{K}_{\mathrm{ir}}$ expression increases between 4 and 7 weeks (Mongiat et al., 2009). In the present study, we show that RMP hyperpolarization occurs steeply as $R_{\text {in }}$ decreases lower than $300 \mathrm{M} \Omega$ (Fig. $3 B$ ), whereas abrupt decrease in firing frequency due to Kv4.1 occurs when $R_{\text {in }}$ decreases lower than $200 \mathrm{M} \Omega$ (Fig. 4G), suggesting that Kv4.1 expression occurs later than GIRK or $\mathrm{K}_{\mathrm{ir}}$ expression, at the very last stage of maturation.

Kv4.1 may play distinctive roles from $\mathrm{K}_{\mathrm{ir}}$ or GIRK channels in lowering the excitability of GCs. We showed that Kv4.1 inhibition increases the gain of $F-I$ curve (Fig. $4 E$ ), whereas inhibition of $\mathrm{K}_{\mathrm{ir}}$ using $\mathrm{BaCl}_{2}$ or GIRK using a GTP-free internal solution shifted the $F-I$ curve to the left with little change in its gain (Mongiat et al., 2009). Considering that $\mathrm{K}_{\mathrm{ir}}$ and GIRK are active at RMP, this difference may suggest that inhibition of background leak currents is capable of reducing threshold currents, but does not affect the gain of input-output relationship significantly. In contrast, Kv4.1 that is activated at depolarized potentials and remains active during repetitive APs (Fig. 6) can regulate the I-O gain.

Brunner et al. (2014) monitored various intrinsic excitability parameters in
A
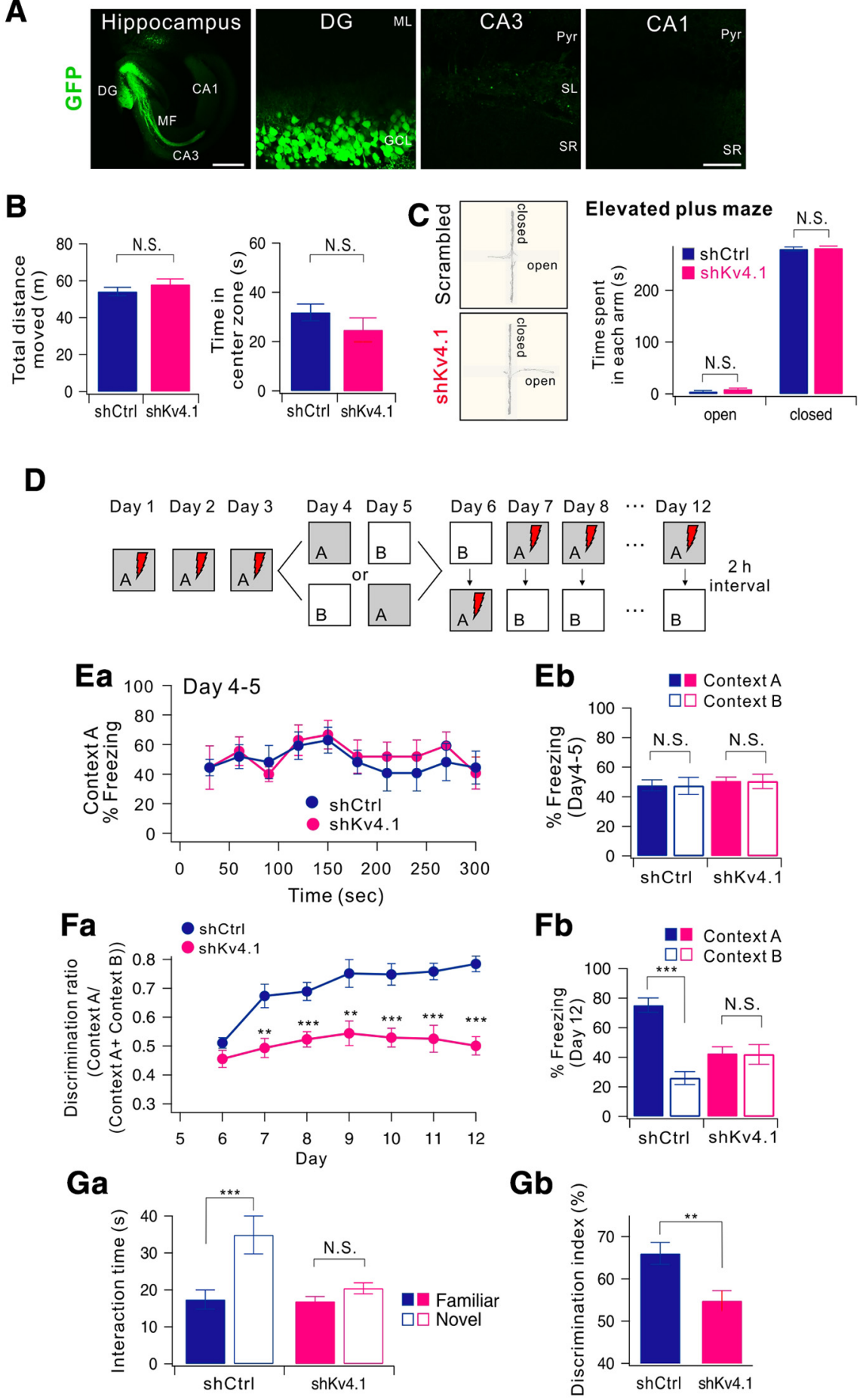

Figure 8. Knock-down of Kv4.1 in DG impairs pattern separation in mice. $\boldsymbol{A}$, Sample images of a 12-week-old mouse that had received a stereotaxic injection of AAV-shKv4.1. GFP expression was restricted to the DG area. Left scale bar, $500 \mu \mathrm{m}$. Right scale bar, $50 \mu \mathrm{m} . B, C, D G$-specific knock-down of Kv4.1 did not affect the general activity and anxiety levels of mice. $B$, Bar graph of total distance traveled for open field box (left) and time spent in the center zone for initial $5 \mathrm{~min}$ (right) in shCtrl-injected (black, $n=9$ ) and shKv4.1-injected mice (red, $n=9), p=0.34$ and 0.34. C, Representative tracking trace of the elevated plus maze (left). Right, Bar graph of time spent in each arm for control (dark blue, $n=9$ ) and shKv4.1-injected mice (pink, $n=9$ ). D, Experimental procedure for pattern separation in 10-week-old mice. Ea, 0 n days 4 to 5 , the kinetics of freezing across the 5 min test in context A. $\boldsymbol{E} \boldsymbol{b}$, Percentage of freezing in context A (filled bars) and context B (open bars) during day 4 to 5 . Control (dark blue, $n=9$ ) and shKv4.1 (pink, $n=9$ ) mice displayed equal amounts of freezing in both contexts A and B. Fa, Time course of the discrimination ratio in control (dark blue, $n=9$ ) and shKv4.1-injected (pink, $n=9$ ) mice during day 6 to 12 . Statistical significance for each day was tested using ANOVA ( ${ }^{*} p<0.05 ;{ }^{* *} p<0.01 ;{ }^{* * *} p<0.001$ ). Fb, Percentage of freezing in context A (filled bars) and context $\mathrm{B}$ (open bars) for control (black, $n=9$ ) and shKv4.1-injected (red, $n=9$ ) mice on day 12. Freezing levels were compared between the two contexts for each group (N.S., no statistical significance; $\left.{ }^{* * *} P=0.000002\right)$. Ga, Time spent interacting with familiar (filled bars) and novel (open bars) objects in shCtrl- $(n=9)$ and shKv4.1-injected $(n=9)$ mice are summarized. Gb, Bar graphs showing the discrimination index of each group for the novel object. 
adult-born GCs from 3 to 10 weeks of age and found that there are two functionally distinct groups in adult-born GCs with respect to the $I-O$ gain. Youngest GCs (3-5 weeks old) are very sensitive to input (S-group, high $I-O$ gain) and oldest GCs (10 weeks old) are less sensitive and respond linearly to input (Lgroup, low $I-O$ gain). Interestingly, the transition from S-group to L-group occurs randomly between 5 and 9 weeks without correlation with the age of the cells or $R_{\text {in }}$ (Brunner et al., 2014). These observations were consistent with the idea that the mechanism responsible for decreasing the input-output gain is different from the mechanism that underlies maturation-dependent decrease in $R_{\text {in }}$ or RMP, but the mechanism is unknown. We provide a line of evidence that Kv4.1 is responsible for the low I-O gain in mature GCs. To our knowledge, our study is the first to identify maturation-dependent changes in voltage-gated $\mathrm{K}^{+}$ channels that can specifically control firing probability.

\section{Distinctive properties of Kv4.1 compared with other Kv4 currents}

Kv4.1 has similar electrophysiological properties to those of Kv4.2 and Kv4.3 when expressed in heterologous expression systems (Pak et al., 1991), showing rapid inactivation kinetics. In a recent study where a role of Kv4.1 in the suprachiasmatic nucleus was suggested, Kv4.1 currents were characterized as A-type $\mathrm{K}^{+}$ currents (Hermanstyne et al., 2017). We found that biophysical properties of Kv4.1 characterized using Kv4.1 antibody in GCs showed a slowly inactivating component (Fig. 6E), which are distinctive from those of classical A-type $\mathrm{K}^{+}$currents. The reason for the observed discrepancy between the biophysical characteristics of $I_{\mathrm{Kv} 4.1}$ in expression systems and GCs is currently unclear. It is plausible that Kv4.1 activity in native neurons might be regulated through diverse modulatory mechanisms which may be absent in in vitro expression systems, such as splice variants of $\mathrm{Kv}$ channel-interacting proteins (KChIPs) that have a transmembrane domain at their $\mathrm{N}$ terminus, also known as transmembrane KChIPs (tmKChIPs), acting as $\mathrm{K}$ channel inactivation suppressor (Holmqvist et al., 2002; Jerng and Pfaffingger, 2008).

We found that there was an obvious contrast between the distribution of Kv4.1 and Kv4.2 (Fig. $1 B, C$ ) in the hippocampus, but the mechanisms and the physiological significance of the distinctive distribution remain to be investigated. In CA1-PCs, $I_{\mathrm{A}}$ was shown to be mediated by Kv4.2, mainly regulating AP repolarization phase (Kim et al., 2005, 2007; Chen et al., 2006). The role of $I_{\mathrm{A}}$ in the regulation of dendritic excitability in GCs was proposed from the experiment using 4 -AP puff $\sim 50 \mu \mathrm{m}$ from the soma (Lopez-Rojas et al., 2016), but the molecular identity of ion channels underlying 4-AP effect remains to be uncovered. Considering that 4-AP can inhibit Kv4.1 as well as Kv4.2, interpretation of 4-AP effects needs to be revisited.

\section{Physiological and pathophysiological implications of Kv4.1 in mature GCs}

It has been postulated that pattern separation reflects nonoverlapping representations of memories on the network (O'Reilly and McClelland, 1994), and thus sparse network activity is prerequisite for pattern separation. Frequency regulation of GCs by Kv4.1 may play a key role in limiting the network activity not only in the DG but also in the CA3 region, since a single MF can induce firing of postsynaptic CA3-PCs only if the inputs are at high frequency (Henze et al., 2002; Bischofberger et al., 2006), whereas low-frequency MF inputs may rather have inhibitory influences to CA3 network via feedforward interneurons (Mori et al., 2004). We also noted that that Kv4.1 is highly expressed also in CA3 (Fig.
$1 A)$, where the network activity is also sparse in vivo (Leutgeb et al., 2004; GoodSmith et al., 2017; Senzai and Buzsaki, 2017). The role of Kv4.1 in CA3-PCs needs to be investigated.

Strong inhibitory inputs to mature GCs are considered as a major mechanism for low excitability of DG (Marín-Burgin et al., 2012; Dieni et al., 2013). It could be thus argued whether knockdown of Kv4.1 also affects excitability of inhibitory neurons, contributing to impaired pattern separation. However, we found that Kv4.1 antibody has no effect on spike frequency of Vgat-positive interneurons in the DG (Fig. $4 J$ ). This observation is compatible with little Kv4.1 mRNA expression in hippocampal interneurons (Serôdio and Rudy, 1998) and weak Kv4.1 immunoreactive signals in Vgat-positive cells (Fig. $4 J c$ ). Thus, our results suggest that impaired pattern separation caused by the knock-down of Kv4.1 mainly stem from abnormally enhanced excitability in mature GCs.

Mice with ablated adult neurogenesis showed impaired pattern separation when stimuli were presented with little spatial separation (Clelland et al., 2009), whereas enhanced adult hippocampal neurogenesis improved pattern separation (Sahay et al., 2011). In addition, a previous study has shown that transgenic mice where output of mature GCs was specifically inhibited exhibited enhanced or normal pattern separation function (Nakashiba et al., 2012). It was thus suggested that pattern separation seems to be mediated mainly by young GCs rather than mature GCs. We, however, showed that hyperexcitability of mature GCs caused by knock-down of Kv4.1 induces impaired pattern separation, implying that the low excitability of mature GCs is critical for pattern separation. Our results do not necessarily mean that mature GCs play a key role in pattern separation. Possibly, hyperexcitability induces greater participation of mature GCs in DG network and hampers young GCs from discriminating similar experiences. A possibility that mature GCs are not passive bystanders but principal performer in DG functions was also proposed (Lopez-Rojas and Kreutz, 2016). Several recent papers indirectly suggest that mature GCs participate in learning and memory formation. Active GCs identified during spatial exploration corresponded to morphologically mature GCs (Diamantaki et al., 2016), and the engram-dentate neurons have electrophysiological characteristics of mature GCs $\left(R_{\text {in }}\right.$ of 100 $\mathrm{M} \Omega$ ) (Ryan et al., 2015). DG network composed of mature GCs and more excitable and plastic young GCs may work together to accomplish pattern separation.

Although little is known about the roles of Kv4.1 in brain functions, possible involvement of Kv4.1 in neuropsychiatric diseases has been noted. Kv4.1 is one of the genes exhibiting a significant number of variants in schizophrenia patients (Piton et al., 2011), and also suggested as a candidate gene for a new neurological syndrome with mental retardation, choreoathetosis, and abnormal behavior which has been mapped to chromosome Xp11 (Reyniers et al., 1999). Combined with our experimental data, Kv4.1 can be a potential target for the intervention of cognitive deficit or other neuropsychiatric diseases.

\section{References}

Baldwin TJ, Tsaur ML, Lopez GA, Jan YN, Jan LY (1991) Characterization of a mammalian cDNA for an inactivating voltage-sensitive $\mathrm{K}^{+}$channel. Neuron 7:471-483.

Beck H, Clusmann H, Kral T, Schramm J, Heinemann U, Elger CE (1997) Potassium currents in acutely isolated human hippocampal dentate granule cells. J Physiol 498:73-85.

Bischofberger J, Engel D, Frotscher M, Jonas P (2006) Timing and efficacy of transmitter release at mossy fiber synapses in the hippocampal network. Pflugers Arch 453:361-372. 
Brunner J, Neubrandt M, Van-Weert S, Andrási T, Kleine Borgmann $F_{\mathrm{B}}$, Jessberger S, Szabadics J (2014) Adult-born granule cells mature through two functionally distinct states. Elife 3:e03104.

Chen X, Yuan LL, Zhao C, Birnbaum SG, Frick A, Jung WE, Schwarz TL, Sweatt JD, Johnston D (2006) Deletion of Kv4.2 gene eliminates dendritic A-type K ${ }^{+}$current and enhances induction of long-term potentiation in hippocampal CA1 pyramidal neurons. J Neurosci 26:12143-12151.

Clelland CD, Choi M, Romberg C, Clemenson GD Jr, Fragniere A, Tyers P, Jessberger S, Saksida LM, Barker RA, Gage FH, Bussey TJ (2009) A functional role for adult hippocampal neurogenesis in spatial pattern separation. Science 325:210-213.

Diamantaki M, Frey M, Berens P, Preston-Ferrer P, Burgalossi A (2016) Sparse activity of identified dentate granule cells during spatial exploration. Elife 5:e20252.

Dieni CV, Nietz AK, Panichi R, Wadiche JI, Overstreet-Wadiche L (2013) Distinct determinants of sparse activation during granule cell maturation. J Neurosci 33:19131-19142.

Gonzalez JC, Epps SA, Markwardt SJ, Wadiche JI, Overstreet-Wadiche L (2018) Constitutive and synaptic activation of GIRK channels differentiates mature and newborn dentate granule cells. J Neurosci 38:6513-6526.

GoodSmith D, Chen X, Wang C, Kim SH, Song H, Burgalossi A, Christian KM, Knierim JJ (2017) Spatial representations of granule cells and mossy cells of the dentate gyrus. Neuron 93:677-690.e5.

Henze DA, Wittner L, Buzsáki G (2002) Single granule cells reliably discharge targets in the hippocampal CA3 network in vivo. Nat Neurosci 5:790-795.

Hermanstyne TO, Granados-Fuentes D, Mellor RL, Herzog ED, Nerbonne JM (2017) Acute knock-down of Kv4.1 regulates repetitive firing rates and clock gene expression in the suprachiasmatic nucleus and daily rhythms in locomotor behavior. eNeuro 4:ENEURO.0377-16.2017.

Hoffman DA, Magee JC, Colbert CM, Johnston D (1997) $\mathrm{K}^{+}$channel regulation of signal propagation in dendrites of hippocampal pyramidal neurons. Nature 387:869-875.

Holmqvist MH, Cao J, Hernandez-Pineda R, Jacobson MD, Carroll KI, Sung MA, Betty M, Ge P, Gilbride KJ, Brown ME, Jurman ME, Lawson D, Silos-Santiago I, Xie Y, Covarrubias M, Rhodes KJ, Distefano PS, An WF (2002) Elimination of fast inactivation in Kv4 A-type potassium channels by an auxiliary subunit domain. Proc Natl Acad Sci U S A 99:1035-1040.

Jerng HH, Pfaffinger PJ (2008) Multiple kv channel-interacting proteins contain an N-terminal transmembrane domain that regulates Kv4 channel trafficking and gating. J Biol Chem 283:36046-36059.

Jinde S, Zsiros V, Jiang Z, Nakao K, Pickel J, Kohno K, Belforte JE, Nakazawa K (2012) Hilar mossy cell degeneration causes transient dentate granule cell hyperexcitability and impaired pattern separation. Neuron 76:1189-1200.

Kerloch T, Clavreul S, Goron A, Abrous DN, Pacary E (2019) Dentate granule neurons generated during perinatal life display distinct morphological features compared with later-born neurons in the mouse hippocampus. Cereb Cortex 29:3527-3539.

Kim J, Wei DS, Hoffman DA (2005) Kv4 potassium channel subunits control action potential repolarization and frequency-dependent broadening in rat hippocampal CA1 pyramidal neurones. J Physiol 569:41-57.

Kim J, Jung SC, Clemens AM, Petralia RS, Hoffman DA (2007) Regulation of dendritic excitability by activity-dependent trafficking of the A-type $\mathrm{K}^{+}$channel subunit Kv4.2 in hippocampal neurons. Neuron 54:933-947.

Laplagne DA, Espósito MS, Piatti VC, Morgenstern NA, Zhao C, van Praag H, Gage FH, Schinder AF (2006) Functional convergence of neurons generated in the developing and adult hippocampus. PLoS Biol 4:e409.

Leutgeb JK, Leutgeb S, Moser MB, Moser EI (2007) Pattern separation in the dentate gyrus and CA3 of the hippocampus. Science 315:961-966.

Leutgeb S, Leutgeb JK, Treves A, Moser MB, Moser EI (2004) Distinct ensemble codes in hippocampal areas CA3 and CA1. Science 305:1295-1298.

Locke RE, Nerbonne JM (1997) Role of voltage-gated $\mathrm{K}^{+}$currents in mediating the regular-spiking phenotype of callosal-projecting rat visual cortical neurons. J Neurophysiol 78:2321-2335.

Lopez-Rojas J, Kreutz MR (2016) Mature granule cells of the dentate gyruspassive bystanders or principal performers in hippocampal function? Neurosci Biobehav Rev 64:167-174.

Lopez-Rojas J, Heine M, Kreutz MR (2016) Plasticity of intrinsic excitability in mature granule cells of the dentate gyrus. Sci Rep 6:21615.

Marín-Burgin A, Mongiat LA, Pardi MB, Schinder AF (2012) Unique processing during a period of high excitation/inhibition balance in adultborn neurons. Science 335:1238-1242.
McHugh TJ, Jones MW, Quinn JJ, Balthasar N, Coppari R, Elmquist JK, Lowell BB, Fanselow MS, Wilson MA, Tonegawa S (2007) Dentate gyrus NMDA receptors mediate rapid pattern separation in the hippocampal network. Science 317:94-99.

McNaughton B, Nadel L (1990) Hebb-Marr networks and the neurobiological representation of action in space. In: Neuroscience and connectionist theory (Gluck M, Rummelhart D, eds), pp 1-63. Mahwah, NJ: Lawrence Erlbaum Associates.

Mongiat LA, Espósito MS, Lombardi G, Schinder AF (2009) Reliable activation of immature neurons in the adult hippocampus. PLoS One 4:e5320.

Mori M, Abegg MH, Gähwiler BH, Gerber U (2004) A frequencydependent switch from inhibition to excitation in a hippocampal unitary circuit. Nature 431:453-456.

Nakamura TY, Nandi S, Pountney DJ, Artman M, Rudy B, Coetzee WA (2001) Different effects of the $\mathrm{Ca}^{2+}$-binding protein, KChIP1, on two Kv4 subfamily members, Kv4.1 and Kv4.2. FEBS Lett 499:205-209.

Nakashiba T, Cushman JD, Pelkey KA, Renaudineau S, Buhl DL, McHugh TJ, Rodriguez Barrera V, Chittajallu R, Iwamoto KS, McBain CJ, Fanselow MS, Tonegawa S (2012) Young dentate granule cells mediate pattern separation, whereas old granule cells facilitate pattern completion. Cell 149:188-201.

O’Reilly RC, McClelland JL (1994) Hippocampal conjunctive encoding, storage, and recall: avoiding a trade-off. Hippocampus 4:661-682.

Pak MD, Baker K, Covarrubias M, Butler A, Ratcliffe A, Salkoff L (1991) mShal, a subfamily of A-type $\mathrm{K}^{+}$channel cloned from mammalian brain. Proc Natl Acad Sci U S A 88:4386-4390.

Piton A, Gauthier J, Hamdan FF, Lafrenière RG, Yang Y, Henrion E, Laurent S, Noreau A, Thibodeau P, Karemera L, Spiegelman D, Kuku F, Duguay J, Destroismaisons L, Jolivet P, Côtó M, Lachapelle K, Diallo O, Raymond A, Marineau C, et al. (2011) Systematic resequencing of X-chromosome synaptic genes in autism spectrum disorder and schizophrenia. Mol Psychiatry 16:867-880.

Ramakers GM, Storm JF (2002) A postsynaptic transient $\mathrm{K}^{+}$current modulated by arachidonic acid regulates synaptic integration and threshold for LTP induction in hippocampal pyramidal cells. Proc Natl Acad Sci U S A 99:10144-10149.

Reyniers E, Van Bogaert P, Peeters N, Vits L, Pauly F, Fransen E, Van Regemorter N, Kooy RF (1999) A new neurological syndrome with mental retardation, choreoathetosis, and abnormal behavior maps to chromosome Xp11. Am J Hum Genet 65:1406-1412.

Rhodes KJ, Carroll KI, Sung MA, Doliveira LC, Monaghan MM, Burke SL, Strassle BW, Buchwalder L, Menegola M, Cao J, An WF, Trimmer JS (2004) KChIPs and Kv4 alpha subunits as integral components of A-type potassium channels in mammalian brain. J Neurosci 24:7903-7915.

Rolls ET (2013) The mechanisms for pattern completion and pattern separation in the hippocampus. Front Syst Neurosci 7:74.

Ryan TJ, Roy DS, Pignatelli M, Arons A, Tonegawa S (2015) Memory: engram cells retain memory under retrograde amnesia. Science 348:1007-1013.

Sahay A, Scobie KN, Hill AS, O'Carroll CM, Kheirbek MA, Burghardt NS, Fenton AA, Dranovsky A, Hen R (2011) Increasing adult hippocampal neurogenesis is sufficient to improve pattern separation. Nature 472:466-470.

Schmidt-Hieber C, Jonas P, Bischofberger J (2004) Enhanced synaptic plasticity in newly generated granule cells of the adult hippocampus. Nature 429:184-187.

Senzai Y, Buzsáki G (2017) Physiological properties and behavioral correlates of hippocampal granule cells and mossy cells. Neuron 93:691-704.e5.

Serôdio P, Rudy B (1998) Differential expression of Kv4 $\mathrm{K}^{+}$channel subunits mediating subthreshold transient $\mathrm{K}^{+}$(A-type) currents in rat brain. J Neurophysiol 79:1081-1091.

Serôdio P, Vega-Saenz de Miera E, Rudy B (1996) Cloning of a novel component of A-type $\mathrm{K}^{+}$channels operating at subthreshold potentials with unique expression in heart and brain. J Neurophysiol 75:2174-2179.

Shibata R, Nakahira K, Shibasaki K, Wakazono Y, Imoto K, Ikenaka K (2000) A-type $\mathrm{K}^{+}$current mediated by the $\mathrm{Kv} 4$ channel regulates the generation of action potential in developing cerebellar granule cells. J Neurosci 20:4145-4155.

Storm JF (1988) Temporal integration by a slowly inactivating $\mathrm{K}^{+}$current in hippocampal neurons. Nature 336:379-381.

Treves A, Rolls ET (1994) Computational analysis of the role of the hippocampus in memory. Hippocampus 4:374-391.

van Praag H, Schinder AF, Christie BR, Toni N, Palmer TD, Gage FH (2002) Functional neurogenesis in the adult hippocampus. Nature 415:1030-1034. 